\title{
Regularity of polyharmonic maps in the critical dimension
}

\author{
Andreas Gastel and Christoph Scheven
}

\begin{abstract}
We prove regularity of weakly $m$-polyharmonic maps (extrinsic or intrinsic) from domains in $\mathbb{R}^{n}$ of dimension $n=2 m \geq 4$ to compact Riemannian manifolds, thus extending a previous result by Wang for the case $m=2$. Moreover, we prove smoothness of Hölder continuous weakly polyharmonic maps for domains in $\mathbb{R}^{n}$ of dimension $n \geq 2 m$.
\end{abstract}

\section{Introduction and statement of results}

Higher order geometric variational problems have attracted quite some attention in recent years. A common feature that makes them interesting for the analyst is the fact that they tend to be associated to systems of higher order partial differential equations with critical growth nonlinearities. For such partial differential equations, regularity of weak solutions is an issue, since we are in a borderline case where classical methods just fail to apply.

For mappings $u: M \rightarrow N$ between Riemannian manifolds, the by now classical variational problem is the one associated to the energy $E(u):=$ $\frac{1}{2} \int_{M}|D u|^{2}$, the critical points of that are harmonic maps. Regularity questions for harmonic maps are quite well understood, in spite of some open questions. In two dimensions (of the domain) the energy is conformally invariant, and harmonic maps are smooth. For higher-dimensional domains only partial regularity holds, and only for harmonic maps that are stationary with respect to variations in the domain. Therefore, in more than two dimensions, minimizing the energy does not seem to be the best choice in order to produce smooth minimizers. This is the main reason why the p-energy $E^{p}(u):=\frac{1}{p} \int_{M}|D u|^{p}$ for $p>1$ has been introduced. Minimizers are $C^{1, \alpha}$ as long as $p \geq n$, but for $p \neq 2$ this cannot be improved to give $C^{\infty}$, due to the non-quadratic growth of the functional. Moreover, the regularity for critical points of $E^{p}$ is still an open problem in the general case.

In order to get more natural variational problems with quadratic growth, higher order functionals seem to be a good choice. There has been a quickly 
growing literature on biharmonic maps for some years now. Biharmonic maps are critical points of the bi-energy $E_{2}(u):=\frac{1}{2} \int_{M}|\Delta u|^{2}$. They come in two different flavors, depending on whether one reads $\Delta u$ as the full Laplacian of mappings $M \rightarrow \mathbb{R}^{K}$ (assuming $N \subset \mathbb{R}^{K}$ ) or as its variant using covariant differentiation also with respect to $N$, i.e., as the tension field of $u$. The critical points of the respective bi-energies are called extrinsically, respectively, intrinsically biharmonic maps. While the intrinsic variant is to be considered more geometric because it does not depend on the embedding $N \hookrightarrow \mathbb{R}^{K}$, it is less natural from the variational point of view, because sequences bounded in energy are not necessarily bounded in $W^{2,2}$. On the other hand, the $W^{2,2}$-norm (for compact domains and targets) can be bounded by the extrinsic bi-energy, which in turn does depend on the embedding of $N$. In any case, bi-energies have good scaling properties on four-dimensional domains.

For biharmonic maps from $\mathbb{R}^{4}$ to a compact Riemannian manifold, both intrinsic and extrinsic, Wang [23] (extending [4]) has proved smoothness. Moreover, he also proves [24] that stationary extrinsically biharmonic maps from $\mathbb{R}^{m}$ are smooth outside a closed singular set of vanishing $(m-4)$ dimensional Hausdorff measure. The second author [17] recently has improved the co-dimension to be at least 5 in the case of minimizers. Lamm and Rivière [12] have given a different proof of continuity of weakly biharmonic maps in four dimensions using a completely different point of view based on conservation laws.

While the bi-energy gives a nice functional on $\mathbb{R}^{4}$, there is a similarly nice one on $\mathbb{R}^{2 m}$, namely the $m$-polyenergy $E_{m}(u):=\frac{1}{2} \int_{\mathbf{R}^{2 m}}\left|D^{m} u\right|^{2}$. Again, there is an extrinsic variant depending on the embedding $N \hookrightarrow \mathbb{R}^{K}$ and an intrinsic one interpreting $D$ as the covariant derivative with respect to both $M$ and $N$. We will restrict ourselves to flat domains $\Omega \subseteq \mathbb{R}^{2 m}$. It should be noted, that on $M$ with curvature, the polyenergy is probably not the most natural choice (nor is the bi-energy) due to the lack of conformal invariance in $2 m$ dimensions. There is a conformally invariant version of the polyenergies which is obtained by replacing $\left|D^{m} u\right|^{2}$ by $\left\langle P_{2 m} u, u\right\rangle$ with the $2 m$ th order Paneitz-type operator. This functional differs from the polyenergy only by lower-order terms, and on $\mathbb{R}^{2 m}$ they coincide. Since we work on $\mathbb{R}^{2 m}$ anyway, we do not have to bother and can work with the polyenergies. See [17] for some reasons why the Paneitz bi-energy should be preferred over the bi-energy.

Since in the extrinsic case, the bi-energy and the 2-polyenergy on $\mathbb{R}^{4}$ differ only by a null Lagrangian, extrinsically biharmonic maps are exactly the extrinsically 2-polyharmonic maps. 
Intrinsically polyharmonic maps have been proposed by Eells and Lemaire [6, Problem (8.8)] who asked about existence results in the critical dimension $2 m$. We will cover regularity results in this dimension for both intrinsically and extrinsically polyharmonic maps, which is a closely related question. Two recent papers have studied regularity issues for (extrinsically) polyharmonic maps. In [7], the corresponding evolution equation is studied for the critical dimension. It is proven that the flow has eternal solutions which develop only finitely many distinct point singularities. There are no singularities at all if the initial polyenergy is below some threshold. In [2], Angelsberg and Pumberger prove that polyharmonic maps (for any domain dimension) are regular at points where $u$ is small in some natural MorreySobolev norm and where for some reason $D^{m} u$ has slightly better integrability than $L^{2}$. The latter condition is a strong one, allowing to make a proof using only growth properties, but basically no structure of the nonlinearities.

In this paper, we are concerned with $2 m$-dimensional domains only. For lower domain dimension, smoothness of $m$-polyharmonic maps can be inferred by standard methods. For higher domain dimension, a variant of Wang's biharmonic proof [24] should be expected to apply. However, there is a major obstacle to trying this, which is the lack of a suitable monotonicity formula for polyharmonic maps. Such a monotonicity formula has been proven by Chang et al. [4], with present form due to Angelsberg [1], for extrinsically biharmonic maps, but their proof does not seem to carry over to the polyharmonic case.

Before stating our main result, let us first give a precise definition of the objects mentioned above. Let $N \subset \mathbb{R}^{K}$ be a smooth compact submanifold. We define the extrinsic and the intrinsic $m$-polyenergy on a domain $\Omega \subset \mathbb{R}^{2 m}$ as

$$
E_{m}(u):=\frac{1}{2} \int_{\Omega}\left|D^{m} u\right|^{2} d x \quad \text { and } \quad \widetilde{E}_{m}(u):=\frac{1}{2} \int_{\Omega}\left|\nabla^{m-1} D u\right|^{2} d x
$$

for all $u \in W^{m, 2}(\Omega, N)$, where $m \geq 2$ and $\nabla$ denotes the Levi-Civita connection on $N$. Here, the space of Sobolev maps with values in $N \subset \mathbb{R}^{K}$ is defined as

$$
W^{m, 2}(\Omega, N):=\left\{u \in W^{m, 2}\left(\Omega, \mathbb{R}^{K}\right): u(x) \in N \text { for almost every } x \in \Omega\right\} \text {. }
$$

We point out that the extrinsic polyenergy depends on the embedding $N \hookrightarrow$ $\mathbb{R}^{K}$, while the intrinsic variant makes sense also for abstract manifolds $N$. 
A map $u \in W^{m, 2}(\Omega, N)$ is called weakly extrinsically (or intrinsically) $m$-polyharmonic if

$$
\left.\frac{d}{d t}\right|_{t=0} E_{m}\left(u_{t}\right)=0, \quad \text { respectively, }\left.\quad \frac{d}{d t}\right|_{t=0} \widetilde{E}_{m}\left(u_{t}\right)=0
$$

holds for all variations $u_{t}:=\pi_{N}(u+t W)$ of $u$, with an arbitrary test vector field $W \in C_{0}^{\infty}\left(\Omega, \mathbb{R}^{K}\right)$. Here, $\pi_{N}$ denotes the nearest-point retraction onto $N$.

We prove the following theorem.

Theorem 1.1 (Regularity in the critical dimension). Let $m \geq 2$ and $\Omega \subset \mathbb{R}^{2 m}$ open, and let $N \subset \mathbb{R}^{K}$ be a smooth compact Riemannian manifold. Then every weakly (extrinsically or intrinsically) m-polyharmonic map $u \in$ $W^{m, 2}(\Omega, N)$ is smooth on $\Omega$.

We point out that in the case $m=2$, this result was established by Wang [23]. Meanwhile, further related results have been accomplished. Lamm and Wang [13] have proven the corresponding boundary regularity theorem for polyharmonic maps with Dirichlet data. Goldstein et al. [9] have pointed out that the special case of $N=S^{n}$ allows a simpler proof without gauge fixing.

For Hölder continuity of $u$, our proof follows Wang's proof [23] for the biharmonic case which is partially based on ideas by Shatah and Struwe [18]. In particular, we use moving frames gauged with the help of Uhlenbeck's gauge theorem [22]. It turns out that Uhlenbeck's gauge is good enough to obey higher order estimates beyond $W^{2,2}$. A suitable formulation of the Euler-Lagrange equation in that frame allows to derive Lorentz space growth estimates quite analogous to Wang's. We avoid Wang's continuation constructions from [23, Lemma 4.3] (which could be difficult to verify) by applying Hodge decomposition to a slightly modified term. Also, compared to Wang's proof, we write the Euler-Lagrange equations for extrinsically and intrinsically polyharmonic maps in a form rather similar to each other, which again allows some simplification.

Once we have Hölder continuity, higher regularity of polyharmonic maps is a matter of methods that have been sketched for the biharmonic case by Chang et al. [4]. However, since this point is not completely trivial (and even less in the polyharmonic case), we think it deserves a more detailed presentation, which we will give in Section 7 . We prove a proposition (see Proposition 7.1) that holds for rather general critical growth p.d.e. and 
also in supercritical dimensions. For polyharmonic maps, it implies as a particular case

Theorem 1.2 (Higher regularity). Let $m \geq 2, \Omega \subseteq \mathbb{R}^{n}(n \geq 2 m)$ be open, and let $N \subset \mathbb{R}^{K}$ be a Riemannian manifold. Then every weakly (extrinsically or intrinsically) m-polyharmonic map $\Omega \rightarrow N$ that is locally Hölder continuous is smooth on $\Omega$.

This theorem also allows to pass from Hölder continuity to smoothness in [2], where the reader is referred to "elliptic bootstrapping arguments".

It should be remarked that existence of weakly polyharmonic maps is accessible in the extrinsic case, where the direct method of the Calculus of Variations produces minimizers in $W^{m, 2}$. In contrast to this, the direct method does not immediately apply to the intrinsic polyenergies. However, Moser [14] recently proved existence of intrinsically polyharmonic maps by minimizing the polyenergy in some variant of $W^{m, 2}$ which is based on intrinsic higher derivatives. While such minimizers are not known to be weak solutions of the polyharmonic map equation in general, for the special case of biharmonic maps in four dimensions, Moser can prove they are. Therefore, including also intrinsically weakly polyharmonic maps in this paper seems to make sense.

\section{Preliminaries on Lorentz spaces}

We will make extensive use of Lorentz spaces and their properties. A good source is Ziemer's book [25, Sections 1.8 and 2.10].

For measurable functions $f: \Omega \rightarrow \mathbb{R}$, we have the nonincreasing rearrangement of $|f|, f^{*}: \mathbb{R}_{+} \rightarrow \mathbb{R}_{+}$with

$$
f^{*}(t):=\inf \{y>0:|\{x \in \Omega:|f(x)|>y\}| \leq t\}
$$

which we use to define

$$
f^{* *}(x):=\frac{1}{x} \int_{0}^{x} f^{*}(t) d t
$$

for $x \geq 0$. For $1<p<\infty$ and $1 \leq q \leq \infty$, the Lorentz space $L^{p, q}(\Omega)$ is the space of functions $f: \Omega \rightarrow \mathbb{R}$ with

$$
\|f\|_{L^{p, q}(\Omega)}:=\left(\int_{0}^{\infty}\left(t^{1 / p} f^{* *}(t)\right)^{q} \frac{d t}{t}\right)^{1 / q}<\infty
$$


if $q<\infty$, or

$$
\|f\|_{L^{p, \infty}(\Omega)}:=\sup _{t>0} t^{1 / p} f^{* *}(t)<\infty .
$$

For $1<p<\infty$ and $1 \leq q_{1}<q_{2} \leq \infty$ we have $L^{p}(\Omega)=L^{p, p}(\Omega), L^{p, q_{1}}(\Omega) \subset$ $L^{p, q_{2}}(\Omega)$, and

$$
\|f\|_{L^{p, q_{2}}(\Omega)} \leq C\|f\|_{L^{p, q_{1}}(\Omega)}
$$

for all $f \in L^{p, q_{1}}(\Omega)$, where the constant $C$ depends only on $p, q_{1}$ and $q_{2}$. For the proofs we refer to the Lemmas 1.8.10 and 1.8.13 in [25].

If $|\Omega|<\infty$, then $L^{p, q}(\Omega) \supset L^{r, s}(\Omega)$ for $1<p<r<\infty, 1 \leq q, s \leq \infty$. In particular, we have the estimate

$$
\|f\|_{L^{p, p}(\Omega)} \leq C_{r, p}|\Omega|^{(1 / p)-(1 / r)}\|f\|_{L^{r, \infty}(\Omega)}
$$

for all $f \in L^{r, \infty}(\Omega)$ and $1<p<r<\infty$. The estimate follows from the definition of the Lorentz spaces by an elementary computation.

Sobolev-Lorentz embedding theorems. The Sobolev embedding theorem can be generalized to the scale of Lorentz spaces as follows. If $f \in$ $W^{k}\left(\mathbb{R}^{n}, \mathbb{R}\right)$ with $D^{k} f \in L^{p, q}\left(\mathbb{R}^{n}\right)$ for some $k \in \mathbb{N}, 1<p<\frac{n}{k}$ and $1 \leq q \leq$ $\infty$, then $f \in L^{n p /(n-k p), q}\left(\mathbb{R}^{n}\right)$ and

$$
\|f\|_{L^{n p /(n-k p), q}\left(\mathbf{R}^{n}\right)} \leq C\left\|D^{k} f\right\|_{L^{p, q}\left(\mathbf{R}^{n}\right)}
$$

A proof can be found in [21]. On balls, the analogous statement holds with the full "Sobolev-Lorentz" norm on the right-hand side,

$$
\|f\|_{L^{n p /(n-k p), q}(B)} \leq C \sum_{j=0}^{k}\left\|D^{j} f\right\|_{L^{p, q}(B)}
$$

This can be checked easily in the case $k=1$ by extending $f$ to a function $\tilde{f} \in W^{1, p}\left(\mathbb{R}^{n}\right)$ with $\|D \tilde{f}\|_{L_{\tilde{f}, q}^{p, q}\left(\mathbf{R}^{n}\right)} \leq C\left(\|f\|_{L^{p, q}(B)}+\|D f\|_{L^{p, q}(B)}\right)$ and applying the estimate $(2.3)$ to $\tilde{f}$. Successive applications of this result then yield the above estimate for all $k \in \mathbb{N}$.

Moreover, we have a Lorentz version of Poincaré's inequality. For $1<$ $p<\infty$, we have

$$
\|f-\bar{f}\|_{L^{p, \infty}(B)} \leq C\|D f\|_{L^{p, \infty}(B)}
$$


for all $f \in W^{1, p}(B)$, where $\bar{f}$ denotes the mean value of $f$ over $B$. To prove this, we use (2.1), the usual Sobolev embedding and (2.2) to find

$$
\|f-\bar{f}\|_{L^{p, \infty}(B)} \leq C\|f-\bar{f}\|_{L^{p}(B)} \leq C\|D f\|_{L^{n p /(n+p)}(B)} \leq C\|D f\|_{L^{p, \infty}(B)} .
$$

Convolution inequalities. In the regularity proofs, convolution inequalities will play a central role. Assume that for $1<p_{1}, p_{2}<\infty$ with $\frac{1}{p_{1}}+\frac{1}{p_{2}}>1$ and $1 \leq q_{1}, q_{2} \leq \infty$, we have $f \in L^{p_{1}, q_{1}}\left(\mathbb{R}^{n}\right)$ and $g \in L^{p_{2}, q_{2}}\left(\mathbb{R}^{n}\right)$. Then there holds

$$
f * g \in L^{r, s}\left(\mathbb{R}^{n}\right) \quad \text { with } \quad\|f * g\|_{L^{r, s}} \leq C\|f\|_{L^{p_{1}, q_{1}}}\|g\|_{L^{p_{2}, q_{2}}}
$$

if $\frac{1}{p_{1}}+\frac{1}{p_{2}}=1+\frac{1}{r}$ and $s \geq 1$ is any number with $\frac{1}{q_{1}}+\frac{1}{q_{2}} \geq \frac{1}{s}$. In the case that we only have $g \in L^{1}\left(\mathbb{R}^{n}\right)$, we have the analogous result

$$
f * g \in L^{p_{1}, q_{1}}\left(\mathbb{R}^{n}\right) \quad \text { with } \quad\|f * g\|_{L^{p_{1}, q_{1}}} \leq C\|f\|_{L^{p_{1}, q_{1}}}\|g\|_{L^{1}}
$$

For the proofs, see [25, Theorem 2.10.1] or [16, Theorem 2.6]. By classical results, Calderon-Zygmund operators map $L^{p, q}\left(\mathbb{R}^{m}\right)$ continuously into itself, see [20, Theorems V.3.15 and VI.3.1]. Therefore, if $K$ is a CalderonZygmund kernel, then

$$
K * g \in L^{p_{2}, q_{2}}\left(\mathbb{R}^{n}\right) \quad \text { with } \quad\|K * g\|_{L^{p_{2}, q_{2}}} \leq C_{K}\|g\|_{L^{p_{2}, q_{2}}}
$$

Multiplication rules. Similarly, we have the following multiplication rules between Lorentz spaces. Assume $f \in L^{p_{1}, q_{1}}\left(\mathbb{R}^{n}\right)$ and $g \in L^{p_{2}, q_{2}}\left(\mathbb{R}^{n}\right)$, where here $1<p_{1}, p_{2}<\infty$ with $\frac{1}{p_{1}}+\frac{1}{p_{2}}<1$ and $1 \leq q_{1}, q_{2} \leq \infty$. Then we have the inequality

$$
\|f g\|_{L^{r, s}} \leq C\|f\|_{L^{p_{1}, q_{1}}}\|g\|_{L^{p_{2}, q_{2}}}
$$

if $\frac{1}{p_{1}}+\frac{1}{p_{2}}=\frac{1}{r}$ and $\frac{1}{q_{1}}+\frac{1}{q_{2}} \geq \frac{1}{s}$ for $s \geq 1$. In the case $\frac{1}{p_{1}}+\frac{1}{p_{2}}=1$, an analogous inequality holds in the sense

$$
\|f g\|_{L^{1}} \leq\|f\|_{L^{p_{1}, q_{1}}}\|g\|_{L^{p_{2}, q_{2}}}
$$

whenever $\frac{1}{q_{1}}+\frac{1}{q_{2}} \geq 1$. For a proof, cf. [16, Theorems 3.4 and 3.5]. Finally, we will need

Lemma 2.1 (Fundamental elliptic inequality for Lorentz norms). On the unit ball $B \subset \mathbb{R}^{n}$ we consider a function $f \in W^{2, p}(B)$ with 
$\Delta f \in L^{p, q}(B)$, where $1<p<\infty$ and $1 \leq q \leq \infty$. Then we have for any $0<r<1$

$$
\left\|D^{2} f\right\|_{L^{p, q}\left(B_{r}\right)} \leq C\left(\|\Delta f\|_{L^{p, q}(B)}+\|D f\|_{L^{p, q}(B)}+\|f\|_{L^{p, q}(B)}\right)
$$

where the constant $C$ depends only on $r, n, p$ and $q$.

Proof. Let $\psi \in C_{\mathrm{cpt}}^{\infty}(B)$ be a cut-off function with $\psi \equiv 1$ on $B_{r}$ and $\|\psi\|_{C^{1}} \leq$ $C=C(r)$. We define $g \in W^{2, p}\left(\mathbb{R}^{n}\right)$ by $g:=\psi f$, which is to be interpreted as zero outside of $B$. By the choice of $\psi$, there holds

$$
|\Delta g| \leq C(|\Delta f|+|D f|+|f|) \quad \text { a.e. on } B \text {. }
$$

By the classical Calderon-Zygmund estimates, see (2.8), we know

$$
\left\|D^{2} f\right\|_{L^{p, q}\left(B_{r}\right)} \leq\left\|D^{2} g\right\|_{L^{p, q}\left(\mathbf{R}^{n}\right)} \leq C\|\Delta g\|_{L^{p, q}\left(\mathbf{R}^{n}\right)} .
$$

Combining the last two estimates, we establish the lemma.

\section{Hodge decomposition on $\mathbb{R}^{n}$ in Lorentz spaces}

Hodge decomposition has been one of the key techniques for regularity proofs even for harmonic maps. What we need are higher order estimates in Lorentz spaces.

Lemma 3.1. Let $\omega \in W^{k, p}\left(\mathbb{R}^{n}, \wedge^{l} \mathbb{R}^{n}\right)$ be an l-form with $\left|D^{k} \omega\right| \in L^{p, q}\left(\mathbb{R}^{n}\right)$, where here $n \geq 2,1 \leq l \leq n, k \in \mathbb{N}_{0}, 1<p<\infty$ and $1 \leq q \leq \infty$. Then there is a decomposition

$$
\omega=d f+d^{*} g \quad \text { with } d^{*} f=0 \text { and } d g=0
$$

for some $f \in W^{k+1, p}\left(\mathbb{R}^{n}, \wedge^{l-1} \mathbb{R}^{n}\right)$ and $g \in W^{k+1, p}\left(\mathbb{R}^{n}, \wedge^{l+1} \mathbb{R}^{n}\right)$. The forms df and $d^{*} g$ are unique and we have the estimates

$$
\begin{aligned}
\left\|D^{k+1} f\right\|_{L^{p, q}} & \leq C\left\|D^{k-1} d^{*} \omega\right\|_{L^{p, q}} \\
\left\|D^{k+1} g\right\|_{L^{p, q}} & \leq C\left\|D^{k-1} d \omega\right\|_{L^{p, q}}
\end{aligned}
$$

for $k \geq 1$, while in the case $k=0$ we have

$$
\|D f\|_{L^{p, q}}+\|D g\|_{L^{p, q}} \leq C\|\omega\|_{L^{p, q}} .
$$


Proof. Uniqueness follows from the result of Iwaniec and Martin, cf. [11, Section 6], which also includes the estimate (3.1) for $p=q$. Inequality (3.1) extends to the scale of Lorentz spaces by a standard interpolation method, see, e.g. [20, Theorem V.3.15]. In order to prove existence, we write $G_{2}$ for the fundamental solution of the Laplace operator and let

$$
f:=G_{2} * d^{*} \omega \text { and } g:=G_{2} * d \omega
$$

This definition implies

$$
d f+d^{*} g=G_{2} *\left(d d^{*} \omega+d^{*} d \omega\right)=G_{2} * \Delta \omega=\omega
$$

and

$$
d^{*} f=G_{2} * d^{*} d^{*} \omega=0 \text { as well as } \quad d g=G_{2} * d d \omega=0 .
$$

For the Lorentz estimate, we calculate at a point $x \in \mathbb{R}^{n}$

$$
D^{k+1} f(x)=\int_{\mathbf{R}^{n}} D^{2} G_{2}(y) D^{k-1} d^{*} \omega(x-y) d y
$$

for $k \geq 1$, and since $D^{2} G_{2}$ is a Calderon-Zygmund kernel, we infer as in $(2.8)$ that

$$
\left\|D^{k+1} f\right\|_{L^{p, q}} \leq C\left\|D^{k-1} d^{*} \omega\right\|_{L^{p, q}}
$$

The Lorentz norm of $D^{k+1} g$ can be estimated analogously.

\section{The Euler-Lagrange equation}

For most of the paper, we will deal with extrinsically polyharmonic maps only. For intrinsically harmonic maps, only few changes are necessary, and we will discuss them in Section 8.

An extrinsically polyharmonic map is easily seen to satisfy $\Delta^{m} u \perp N$. We will start by deriving a weak formulation of the Euler-Lagrange equation. We can reformulate the above orthogonality relation as

$$
\int\left\langle D^{m} u, D^{m}(\Pi(u) V)\right\rangle d x=0
$$

for every vectorfield $V \in C_{\mathrm{cpt}}^{\infty}\left(\Omega, \mathbb{R}^{K}\right)$, where $\Pi(y): \mathbb{R}^{K} \rightarrow \mathrm{T}_{y} N$ denotes the orthogonal projection. Applying Leibniz' rule in the above equation, we 
compute

$$
\int\left\langle D^{m} u, \Pi(u) D^{m} V\right\rangle d x=-\sum_{k=0}^{m-1}\left(\begin{array}{c}
m \\
k
\end{array}\right) \int\left\langle D^{m} u, D^{m-k}(\Pi(u)) D^{k} V\right\rangle d x .
$$

On the other hand, we have

$$
\begin{aligned}
\int\left\langle D^{m} u, D^{m} V\right\rangle d x= & \int\left\langle D^{m-1}(\Pi(u) D u), D^{m} V\right\rangle d x \\
= & \sum_{k=0}^{m-2}\left(\begin{array}{c}
m-1 \\
k
\end{array}\right) \int\left\langle D^{m-1-k}(\Pi(u)) D^{k+1} u, D^{m} V\right\rangle d x \\
& +\int\left\langle\Pi(u) D^{m} u, D^{m} V\right\rangle d x .
\end{aligned}
$$

Combining the last two equations, we arrive at

$$
\begin{aligned}
\int\left\langle D^{m} u, D^{m} V\right\rangle d x= & \sum_{k=0}^{m-2}\left(\begin{array}{c}
m-1 \\
k
\end{array}\right) \int\left\langle D^{m-1-k}(\Pi(u)) D^{k+1} u, D^{m} V\right\rangle d x \\
& -\sum_{k=0}^{m-1}\left(\begin{array}{c}
m \\
k
\end{array}\right) \int\left\langle D^{m} u, D^{m-k}(\Pi(u)) D^{k} V\right\rangle d x .
\end{aligned}
$$

By approximation, this holds for every $V \in W_{0}^{m, 2} \cap L^{\infty}\left(\Omega, \mathbb{R}^{K}\right)$. Any $u \in$ $W^{m, 2}(\Omega, N)$ satisfying (4.2) will be called a weakly extrinsically $(m-)$ polyharmonic map. This weak form of the Euler-Lagrange equation is a rather simplified version of [2, Lemma 2.2].

To make proper use of the structure of the equation, we will make use of another reformulation that allows to read the equation "in coordinates adapted to $u$ ". Let $u: \Omega \rightarrow N$ be a map of class $W^{m, 2}$, and $\eta: \Omega \rightarrow \mathbb{R}^{K}$ a vectorfield along $u$, i.e., $\eta(x) \in T_{u(x)} N$ for almost all $x \in \Omega$. We have

$$
\Delta\langle\Delta u, \eta\rangle=\left\langle\Delta^{2} u, \eta\right\rangle+\langle D \Delta u, D \eta\rangle+\operatorname{div}\langle\Delta u, D \eta\rangle
$$

which we can iterate to get

$$
\begin{aligned}
\Delta^{m-1}\langle\Delta u, \eta\rangle= & \left\langle\Delta^{m} u, \eta\right\rangle+\sum_{k=1}^{m-1}\left[\Delta^{m-k-1}\left\langle D \Delta^{k} u, D \eta\right\rangle\right. \\
& \left.+\Delta^{m-k-1} \operatorname{div}\left\langle\Delta^{k} u, D \eta\right\rangle\right]
\end{aligned}
$$


by induction. This implies

$$
\Delta^{m-1} \operatorname{div}\langle D u, \eta\rangle=\left\langle\Delta^{m} u, \eta\right\rangle+\Delta^{m-1}\langle D u, D \eta\rangle
$$

$$
+\sum_{k=1}^{m-1}\left[\Delta^{m-k-1}\left\langle D \Delta^{k} u, D \eta\right\rangle+\Delta^{m-k-1} \operatorname{div}\left\langle\Delta^{k} u, D \eta\right\rangle\right]
$$

Iterating

$$
\langle\operatorname{div} v, f\rangle=\operatorname{div}\langle v, f\rangle-\langle v, D f\rangle
$$

we find

$$
\left\langle\operatorname{div}^{k} v, f\right\rangle=\sum_{l=0}^{k}(-1)^{k-l}\left(\begin{array}{l}
k \\
l
\end{array}\right) \operatorname{div}^{l}\left\langle v, D^{k-l} f\right\rangle .
$$

Plugging that into (4.3), we find

$$
\begin{aligned}
\Delta^{m-1} \operatorname{div}\langle D u, \eta\rangle= & \left\langle\Delta^{m} u, \eta\right\rangle+\Delta^{m-1}\langle D u, D \eta\rangle \\
& +\sum_{k=1}^{m-1} \sum_{l=0}^{k}(-1)^{k-l}\left(\begin{array}{l}
k \\
l
\end{array}\right)\left[\Delta^{m-k-1} \operatorname{div}^{l}\left\langle D^{k+1} u, D^{k-l+1} \eta\right\rangle\right. \\
& \left.+\Delta^{m-k-1} \operatorname{div}^{l+1}\left\langle D^{k} u, D^{k-l+1} \eta\right\rangle\right] .
\end{aligned}
$$

Here, all derivatives of order $>m$ are to be interpreted as distributional derivatives. This is no problem because of the divergence structure of the above terms. Now we assume that $u$ is weakly extrinsically $m$-polyharmonic. This is equivalent to the first term on the right-hand side vanishing for all $\eta \in W_{0}^{m, 2} \cap L^{\infty}\left(\Omega, \mathbb{R}^{K}\right)$ that are tangential along $u$.

Thus, we have derived the Euler-Lagrange equation for extrinsically polyharmonic maps in the following form: for all $\eta \in W_{0}^{m, 2} \cap L^{\infty}\left(\Omega, \mathbb{R}^{K}\right)$ tangential along $u$, we have

$$
\begin{aligned}
\Delta^{m-1} \operatorname{div}\langle D u, \eta\rangle= & \Delta^{m-1}\langle D u, D \eta\rangle \\
& +\sum_{k=1}^{m-1} \sum_{l=0}^{k}(-1)^{k-l}\left(\begin{array}{l}
k \\
l
\end{array}\right)\left[\Delta^{m-k-1} \operatorname{div}^{l}\left\langle D^{k+1} u, D^{k-l+1} \eta\right\rangle\right. \\
& \left.+\Delta^{m-k-1} \operatorname{div}^{l+1}\left\langle D^{k} u, D^{k-l+1} \eta\right\rangle\right] .
\end{aligned}
$$

This form of the equation turns out to be useful when $\eta$ stands for the elements of some frame of $u^{*} \mathrm{~T} N$ adapted to $u$, which we will construct in the section that follows. 


\section{Construction of a Coulomb frame along $W^{m, 2}$-maps}

This section adds higher order estimates to the arguments from Section 3 of [23] which is based on Section 4 of [18].

For the construction of the Coulomb frames, we will apply Uhlenbeck's theorem and combine it with the higher order estimates of the following

Lemma 5.1. Let $u \in W^{m, 2}(B, N)$ satisfy $\|D u\|_{W^{m-1,2}} \leq \kappa$ for some $\kappa \in$ $(0,1)$. Assume further that for an orthonormal frame $\left\{e_{\alpha}\right\}_{\alpha=1}^{n} \subset W^{2, m}$ $(B, T N)$ along $u$ and the connection form $A:=\left(\left\langle e_{\alpha}, d e_{\beta}\right\rangle\right)_{\alpha, \beta}$, there holds

$$
d^{*} A=0 \quad \text { and } \quad\|A\|_{W^{1, m}(B)}+\sum_{\alpha=1}^{n}\left\|D e_{\alpha}\right\|_{W^{1, m}(B)} \leq \kappa
$$

for some $\kappa \in(0,1)$. Then we have the estimate

$$
\sum_{j=0}^{m}\left\|D^{j} A\right\|_{L^{2 m /(j+1), 1}\left(B_{1 / 2}\right)} \leq C \kappa
$$

with a constant $C$ depending only on $m$ and $N$.

The proof relies on the following two lemmas. We recall that the curvature of a connection form is defined by $F(A):=d A+[A, A]$.

Lemma 5.2. Let $0 \leq k \leq m-1$ and assume that $A \in W^{k, 2 m /(k+1)}(B$, $\left.\wedge^{1} \mathbb{R}^{2 m} \otimes \mathfrak{s o}(\operatorname{dim} N)\right)$ satisfies $d^{*} A=0$ on $B$ and $\left|D^{k} F(A)\right| \in L^{2 m /(k+2), 1}(B)$. Then for every $0<r<1$ there holds $\left|D^{k+1} A\right| \in L^{2 m /(k+2), m /(k+1)}\left(B_{r}\right)$ with

$$
\begin{aligned}
& \left\|D^{k+1} A\right\|_{L^{2 m /(k+2), m /(k+1)}\left(B_{r}\right)} \\
& \quad \leq C\left(\left\|D^{k} F(A)\right\|_{L^{2 m /(k+2), 1}}+\|A\|_{W^{k, 2 m /(k+1)}}^{2}+\|A\|_{W^{k, 2 m /(k+1)}}\right) .
\end{aligned}
$$

Here, the constant $C$ depends only on $m$ and $r$.

Proof. Since $d^{*} A=0$ and by the definition of $F(A)$, we have

$$
\left|D^{k-1} \Delta A\right| \leq C\left|D^{k} d A\right| \leq C\left|D^{k} F(A)\right|+C\left|D^{k}[A, A]\right|
$$


For the last term, we have the estimate

$$
\begin{aligned}
& \left\|D^{k}[A, A]\right\|_{L^{2 m /(k+2), m /(k+1)}} \\
& \quad \leq C \sum_{i=0}^{k}\left\|D^{i} A\right\|_{L^{2 m /(i+1), 2 m /(k+1)}}\left\|D^{k-i} A\right\|_{L^{2 m /(k-i+1), 2 m /(k+1)}} \\
& \quad \leq C\|A\|_{W^{k, 2 m /(k+1)}}^{2}
\end{aligned}
$$

by the multiplication rule (2.9) and the Sobolev embedding (2.4). We conclude

$$
\left\|\Delta D^{k-1} A\right\|_{L^{2 m /(k+2), m /(k+1)}} \leq C\left(\left\|D^{k} F(A)\right\|_{L^{2 m /(k+2), 1}}+\|A\|_{W^{k, 2 m /(k+1)}}^{2}\right) .
$$

From this, the claim follows by the fundamental elliptic estimate in Lorentz spaces, see Lemma 2.1.

Lemma 5.3. Assume that $\left\{e_{\alpha}\right\}_{\alpha=1}^{\operatorname{dim} N}$ is an orthonormal frame along $u \in$ $W^{m, 2}(B, N)$ and that $A=\left(\left\langle e_{\alpha}, d e_{\beta}\right\rangle\right)$ satisfies $A \in W^{k-1,2 m / k}$ for some $1 \leq$ $k \leq m$. Then $e_{\alpha} \in W^{k, 2 m / k}\left(B, \mathbb{R}^{K}\right)$ for all $1 \leq \alpha \leq \operatorname{dim} N$, and we have the following pointwise estimates on $B$ :

$$
\left|D^{k} e_{\alpha}\right| \leq C \sum_{j=1}^{k}\left(\left|D^{j-1} A\right|+\left|D^{j} u\right|\right)^{k / j}
$$

for all $1 \leq \alpha \leq \operatorname{dim} N$. In particular, we have for every $1 \leq p \leq \infty$

$$
\sum_{\alpha=1}^{n}\left\|D^{k} e_{\alpha}\right\|_{L^{2 m / k, p}} \leq C \sum_{l=1}^{k}\left(\left\|D^{l-1} A\right\|_{L^{2 m / l, p k / l}}+\left\|D^{l} u\right\|_{L^{2 m / l, p k / l}}\right)^{k / l}
$$

Proof. The pointwise estimates are a consequence of the identity

$$
D e_{\alpha}=\sum_{\beta=1}^{n}\left\langle D e_{\alpha}, e_{\beta}\right\rangle e_{\beta}+(I \circ u)\left(D u, e_{\alpha}\right) \quad \text { for } 1 \leq \alpha \leq \operatorname{dim} N
$$

which readily implies (5.3) in the case $k=1$. Here, II denotes the second fundamental form of the embedding $N \hookrightarrow \mathbb{R}^{K}$. Now we assume (5.3) for all $1 \leq k<l$ up to some $l \in\{1, \ldots, m\}$, and differentiate the above identity in 
order to get

$$
\begin{aligned}
\left|D^{l} e_{\alpha}\right| \leq & C \sum_{k=0}^{l-1} \sum_{\beta}\left|D^{l-k-1} A\right|\left|D^{k} e_{\beta}\right| \\
& +C \sum_{\substack{i+j+k=l-1 \\
i, j, k \geq 0}} \sum_{h=1}^{i}\left|D^{h} u\right|^{i / h}\left|D^{j+1} u\right|\left|D^{k} e_{\alpha}\right| .
\end{aligned}
$$

Applying Young's inequality, we arrive at

$$
\left|D^{l} e_{\alpha}\right| \leq C \sum_{j=1}^{l}\left(\left|D^{j-1} A\right|^{l / j}+\left|D^{j} u\right|^{l / j}\right)+C \sum_{k=1}^{l-1} \sum_{\beta}\left|D^{k} e_{\beta}\right|^{l / k}
$$

Using the assumption (5.3) for $1 \leq k \leq l-1$, we establish the estimate (5.3) for $k=l$. The Lorentz norm estimate is an immediate consequence of $(5.3)$.

Proof of Lemma 5.1. First of all, note that the assumption (5.1) and the properties of $u$ imply by the Sobolev embedding theorem

$$
\|A\|_{L^{2 m}(B)}+\sum_{\alpha=1}^{\operatorname{dim} N}\left\|D e_{\alpha}\right\|_{L^{2 m}(B)}+\sum_{k=1}^{m}\left\|D^{k} u\right\|_{L^{2 m / k}(B)} \leq C \kappa .
$$

The curvature of $A$ satisfies

$$
F(A)_{\alpha \beta}=\left\langle\left(R^{N} \circ u\right)(d u \wedge d u) e_{\beta}, e_{\alpha}\right\rangle \text { on } B
$$

with the Riemannian curvature tensor $R^{N}$ on $N$, cf. [23, Section 3]. This implies in particular, using (2.9) and (2.4),

$$
\|F(A)\|_{L^{m, 1}(B)} \leq C\|D u\|_{L^{2 m, 2}(B)}^{2} \leq C\|D u\|_{W^{m-1,2}(B)}^{2} \leq C \kappa^{2} .
$$

For $2 \leq k \leq m+1$ we let $r_{k}:=2^{(2-k) /(m-1)}$ and $D_{k}:=B_{r_{k}}(0)$, so that $B_{1 / 2}=D_{m+1} \subsetneq D_{m} \subsetneq \cdots \subsetneq D_{2}=B$. By induction, we will prove that $A \in W^{k-1,2 m / k}\left(D_{k}, \wedge^{1} \mathbb{R}^{2 m} \otimes \mathfrak{s o}(n)\right)$ and $e_{\alpha} \in W^{k, 2 m / k}\left(D_{k}, \mathbb{R}^{K}\right)$ for all 
$1 \leq \alpha \leq n$ and $2 \leq k \leq m$ with the estimate

$$
\left\|D^{k-2} F(A)\right\|_{L^{2 m / k, 1}\left(D_{k}\right)}+\left\|D^{k-1} A\right\|_{L^{2 m / k}\left(D_{k}\right)}+\sum_{\alpha=1}^{n}\left\|D^{k} e_{\alpha}\right\|_{L^{2 m / k}\left(D_{k}\right)} \leq C \kappa .
$$

For $k=2$, this follows from (5.6) and the assumption (5.1). Now assume that we know (5.7) for all $2 \leq k \leq l$ up to some $l \in\{2, \ldots, m-1\}$. Identity (5.5) implies, by the chain rule and Young's inequality,

$$
\begin{aligned}
\left|D^{l-1} F(A)_{\alpha \beta}\right| \leq & C \sum_{\substack{i_{1}+\ldots+i_{5}=l-1 \\
i_{j} \geq 0}} \max \left\{1, \sum_{j=1}^{i_{1}}\left|D^{j} u\right|^{i_{1} / j}\right\} \\
& \times\left|D^{i_{2}+1} u\right|\left|D^{i_{3}+1} u\right|\left|D^{i_{4}} e_{\beta}\right|\left|D^{i_{5}} e_{\alpha}\right| .
\end{aligned}
$$

Applying the multiplication rule (2.9) repeatedly, we conclude

$$
\begin{aligned}
\left\|D^{l-1} F(A)\right\|_{L^{2 m /(l+1), 1}\left(D_{l}\right)} \leq & C\left(1+\sum_{i=1}^{l}\left\|D^{i} u\right\|_{L^{2 m / i}}\right) \\
& \times\left(\sum_{i=1}^{l}\left\|D^{i} u\right\|_{L^{2 m / i, 2}}\right)^{2}\left(1+\sum_{j=1}^{l-1} \sum_{\alpha}\left\|D^{j} e_{\alpha}\right\|_{L^{2 m / j}}\right)^{2} \\
\leq & C(1+\kappa)^{3}\|D u\|_{W^{m-1,2}\left(D_{l}\right)}^{2} \leq C \kappa^{2} .
\end{aligned}
$$

Here we used the Sobolev-Lorentz embedding $W^{m-i, 2} \hookrightarrow L^{2 m / i, 2}$. Applying Lemma 5.2 on the ball $D_{l}$, we get $A \in W^{l, 2 m /(l+1)}\left(D_{l+1}\right)$ with

$$
\begin{aligned}
\left\|D^{l} A\right\|_{L^{2 m /(l+1), m / l}\left(D_{l+1}\right)} \leq & C\left(\left\|D^{l-1} F(A)\right\|_{L^{2 m /(l+1), 1}\left(D_{l}\right)}\right. \\
& \left.+\|A\|_{W^{l-1,2 m / l}\left(D_{l}\right)}^{2}+\|A\|_{W^{l-1,2 m / l}\left(D_{l}\right)}\right) \leq C \kappa
\end{aligned}
$$

by the assumption (5.1) and by (5.7) for $k=2, \ldots, l$. This implies by Lemma 5.3

$$
\begin{aligned}
& \left\|D^{l+1} e_{\alpha}\right\|_{L^{2 m /(l+1)}\left(D_{l+1}\right)} \\
& \quad \leq C \sum_{j=1}^{l+1}\left(\left\|D^{j-1} A\right\|_{L^{2 m / j}\left(D_{l+1}\right)}^{(l+1) / j}+\left\|D^{j} u\right\|_{L^{2 m / j}\left(D_{l+1}\right)}^{(l+1) / j}\right) \leq C \kappa
\end{aligned}
$$

for all $1 \leq \alpha \leq n$, by (5.4) and (5.7) for $k=2, \ldots, l$. Estimates (5.8) to (5.10) yield the claim (5.7) for $k=l+1$. In this manner, we can establish 
the estimate (5.7) successively for all $2 \leq k \leq m$. This enables us to apply Lemma 5.2 with $k=m-1$ with the result

$$
\left\|D^{m} A\right\|_{L^{2 m /(m+1), 1}\left(D_{m+1}\right)} \leq C \kappa .
$$

Combining this with (5.4) and (5.7) for $2 \leq k \leq m$, the Sobolev-Lorentz embedding theorem yields the desired estimate (5.2).

Now we are able to prove the main theorem of this section.

Theorem 5.1 (Coulomb gauge). There are constants $\varepsilon_{1} \in(0,1)$ and $C_{1} \in \mathbb{R}$, both depending only on $m$ and $N$, such that for every map $u \in$ $W^{m, 2}(B, N)$ with $\|D u\|_{W^{m-1,2}} \leq \varepsilon_{1}$ there is an orthonormal frame $\left\{e_{\alpha}\right\}_{\alpha=1}^{\operatorname{dim} N}$ $\subset W^{m, 2}\left(B_{1 / 4}, T N\right)$ with $e_{\alpha}(x) \in T_{u(x)} N$ for a.e. $x \in B_{1 / 4}$ such that the connection form $A=\left(\left\langle e_{\alpha}, d e_{\beta}\right\rangle\right)_{\alpha, \beta}$ satisfies

$$
d^{*} A=0 \text { on } B \quad \text { and } \quad \sum_{k=0}^{m}\left\|D^{k} A\right\|_{L^{2 m /(k+1), 1}\left(B_{1 / 4}\right)} \leq C_{1}\|D u\|_{W^{m-1,2}(B)} .
$$

Proof. By arguments due to Schoen and Uhlenbeck [19] we can find an approximating sequence $u_{i} \in C^{\infty}(B, N)$ with $u_{i} \rightarrow u$ strongly in $W^{2, m}$ $\left(B, \mathbb{R}^{K}\right)$ and almost everywhere as $i \rightarrow \infty$. We reproduce the proof for the reader's convenience. Let $\phi \in C_{\mathrm{cpt}}^{\infty}\left(B, \mathbb{R}_{\geq 0}\right)$ be a mollifying kernel with $\|\phi\|_{L^{1}}=1$ and let $\phi_{i}(x):=i^{2 m} \phi(i x)$ for $x \in \mathbb{R}^{2 m}$ and $i \in \mathbb{N}$. We define $\tilde{u}_{i}:=$ $\phi_{i} * u \in C^{\infty}\left(\mathbb{R}^{2 m}, \mathbb{R}^{K}\right)$, where we have extended $u$ by zero outside of $B$ for the definition. Then it holds $\tilde{u}_{i} \rightarrow u$ in $W^{2, m}\left(B_{3 / 4}, \mathbb{R}^{K}\right)$, in $W^{1,2 m}\left(B_{3 / 4}, \mathbb{R}^{K}\right)$ and almost everywhere, as $i \rightarrow \infty$. Moreover, for $x \in B_{3 / 4}$, with the notation $u_{x ; i}:=f_{B_{1 / i}(x)} u(y) d y$, we have

$$
\begin{aligned}
\operatorname{dist}\left(\tilde{u}_{i}(x), N\right) & \leq f_{B_{1 / i}(x)}\left|\tilde{u}_{i}(x)-u(y)\right| d y \\
& \leq \int_{B_{1 / i}(x)} \phi_{i}(x-z) f_{B_{1 / i}(x)}|u(z)-u(y)| d y d z \\
& \leq C f_{B_{1 / i}(x)}\left|u(y)-u_{x ; i}\right| d y \leq C \int_{B_{1 / i}(x)}|D u|^{2 m} d y \leq C \varepsilon_{1}^{2 m}
\end{aligned}
$$

by the Sobolev embedding. Thus, if $\varepsilon_{1}$ is chosen sufficiently small depending on $N$, we can ensure that $u_{i}:=\pi_{N} \circ \tilde{u}_{i} \in C^{\infty}\left(B_{3 / 4}, N\right)$ is well defined, where here $\pi_{N}$ denotes the nearest-point retraction onto $N$. It is straightforward to check that $u_{i} \rightarrow u$ strongly in $W^{2, m}\left(B_{3 / 4}, \mathbb{R}^{K}\right)$ and almost everywhere, as $i \rightarrow \infty$. 
Now we consider the pulled back bundle $u_{i}^{*} T N$, which is a trivial vector bundle since the base space $B_{3 / 4}$ is contractible. This means that we can choose a smooth orthonormal frame $\left\{\tilde{e}_{\alpha}^{i}\right\}_{\alpha=1}^{\operatorname{dim}_{\alpha} N} \subset \Gamma\left(u_{i}^{*} T N\right)$. We want to apply Uhlenbeck's gauge theorem to the connection form $\widetilde{A}^{i}:=\left(\left\langle\tilde{e}_{\alpha}^{i}, d \tilde{e}_{\beta}^{i}\right\rangle\right)_{\alpha, \beta}$, whose curvature satisfies, cf. [23, Section 3],

$$
\left|F\left(\widetilde{A}^{i}\right)\right|=\left|\left(R^{N} \circ u_{i}\right)\left(d u_{i} \wedge d u_{i}\right)\right| \leq C(N)\left|D u_{i}\right|^{2}
$$

where here $R^{N}$ denotes the Riemannian curvature tensor associated with $N$. In particular, with $\varepsilon:=\|D u\|_{W^{m-1,2}(B)}$,

$$
\left\|F\left(\widetilde{A}^{i}\right)\right\|_{L^{m}} \leq C(m, N)\left\|D u_{i}\right\|_{L^{2 m}}^{2} \leq C(m, N) \varepsilon^{2} \leq C(m, N) \varepsilon_{1}^{2}
$$

if $i \in \mathbb{N}$ is chosen sufficiently large. Thus, choosing $\varepsilon_{1}>0$ small depending on $m$ and $N$, Theorem 1.3 of [22] yields a Gauge transformation $s \in$ $W^{2, m}\left(B_{1 / 2}, \mathrm{SO}(\operatorname{dim} N)\right)$ such that the connection forms $A^{i}=\left(\left\langle e_{\alpha}^{i}, d e_{\beta}^{i}\right\rangle\right)$, taken with respect to the frames $e_{\alpha}^{i}:=\sum_{\beta} \tilde{e}_{\beta}^{i} s_{\beta \alpha}$, satisfy

$$
d^{*} A^{i}=0 \text { on } B_{1 / 2} \quad \text { and } \quad\left\|A^{i}\right\|_{W^{1, m}\left(B_{1 / 2}\right)} \leq C \varepsilon^{2}
$$

where the constant $C$ depends only on $m$ and $N$. From Lemma 5.3, we infer

$$
\begin{aligned}
\left\|D e_{\alpha}^{i}\right\|_{W^{1, m}\left(B_{1 / 2}\right)} \leq & C\left(\left\|A^{i}\right\|_{W^{1, m}\left(B_{1 / 2}\right)}+\left\|A^{i}\right\|_{L^{2 m}\left(B_{1 / 2}\right)}^{2}\right. \\
& \left.+\left\|D u_{i}\right\|_{W^{1, m}\left(B_{1 / 2}\right)}+\left\|D u_{i}\right\|_{L^{2 m}\left(B_{1 / 2}\right)}^{2}\right) \\
\leq & C \varepsilon
\end{aligned}
$$

for all $1 \leq \alpha \leq n$ and all sufficiently large $i \in \mathbb{N}$. Here we used the Sobolev embedding and (5.12). The last two estimates ensure that after extracting a subsequence of $\{i\}$, we have convergence $e_{\alpha}^{i} \rightarrow e_{\alpha}$ weakly in $W^{2, m}$, strongly in $W^{1, m}$ and almost everywhere, as well as $A^{i} \rightarrow A$ weakly in $W^{1, m}$ and strongly in $L^{m}$, for some vector fields $e_{\alpha} \in W^{2, m}\left(B_{1 / 2}, \mathbb{R}^{K}\right)$ and a connection form $A \in W^{1, m}\left(B_{1 / 2}, \wedge^{1} \mathbb{R}^{2 m} \otimes \mathfrak{s o}(\operatorname{dim} N)\right)$. By the convergence almost everywhere, we know that $\left\{e_{\alpha}(x)\right\}_{\alpha}$ is an orthonormal basis of $\mathrm{T}_{u(x)} N$ for a.e. $x \in B_{1 / 2}$. Moreover $A=\left(\left\langle e_{\alpha}, d e_{\beta}\right\rangle\right)$, and by (5.12) and (5.13), we have

$$
d^{*} A=0 \text { on } B_{1 / 2} \quad \text { and } \quad\|A\|_{W^{1, m}\left(B_{1 / 2}\right)}+\sum_{\alpha=1}^{n}\left\|D e_{\alpha}\right\|_{W^{1, m}\left(B_{1 / 2}\right)} \leq C \varepsilon
$$

where we used the lower semicontinuity of the norms with respect to weak convergence. The claimed estimate (5.11) now follows from Lemma 5.1. 


\section{Decay estimates}

In this section, we use Lorentz spaces, Hodge decomposition and Coulomb frames to prove

Proposition 6.1 (Decay estimates). There exist $\varepsilon_{0}>0$ and $\theta_{0} \in\left(0, \frac{1}{4}\right)$, such that for any $r>0$ and any weakly extrinsically (or intrinsically) polyharmonic map $u \in W^{m, 2}\left(B_{2 r}, N\right)$ satisfying

$$
\sum_{j=1}^{m}\left\|D^{j} u\right\|_{L^{2 m / j}\left(B_{4 r}\right)} \leq \varepsilon_{0}
$$

we have the estimate

$$
\sum_{j=1}^{m}\left\|D^{j} u\right\|_{L^{2 m / j, \infty}\left(B_{\theta_{0} r}\right)} \leq \frac{1}{2} \sum_{j=1}^{m}\left\|D^{j} u\right\|_{L^{2 m / j, \infty}\left(B_{r}\right)} .
$$

Proof. We will give the proof for the extrinsic case here and will discuss the necessary changes for the intrinsic case in Section 8.

We can assume $r=1$. We choose a Coulomb frame $\left\{e_{i}\right\}_{i=1}^{n} \subset W^{m, 2}$ $(B, \mathrm{~T} N)$ along $u$ as in Theorem 5.1 and set $A_{i j}:=\left\langle e_{i}, d e_{j}\right\rangle$. The same theorem yields the estimates

$$
\sum_{j=1}^{m}\left\|D^{j-1} A\right\|_{L^{2 m / j, 1}(B)} \leq C\|D u\|_{W^{m-1,2}\left(B_{4}\right)} \leq C \varepsilon_{0}
$$

as long as we have chosen $\varepsilon_{0}$ sufficiently small. In what follows, we fix $i \in$ $\{1, \ldots, n\}$. Let $\varphi \in C_{\mathrm{cpt}}^{\infty}(B)$ be a cut-off function with $\varphi \equiv 1$ on $B_{1 / 2}$ and $\|\varphi\|_{C^{m}} \leq C$.

We perform a Hodge decomposition

$$
\left\langle d(\varphi(u-\bar{u})), e_{i}\right\rangle=d f_{i}+d^{*} g_{i} \quad \text { with } d g_{i}=0
$$

according to Lemma 3.1. Here, $\bar{u}$ denotes the mean value of $u$ over $B$. Applying $\Delta^{m-1} d^{*}$ to the above decomposition, we find

$$
\Delta^{m} f_{i}=\Delta^{m-1} \operatorname{div}\left\langle D u, e_{i}\right\rangle \quad \text { on } B_{1 / 2}
$$

because $\varphi \equiv 1$ holds there. 
For $k=0, \ldots, m-1$ and $l=0, \ldots, k$, we define $\omega_{k, l}^{i}$ and $\eta_{k, l}^{i}$ to be the solutions of

$$
\begin{aligned}
& \Delta^{m} \omega_{k, l}^{i}=(-1)^{k-l}\left(\begin{array}{c}
k \\
l
\end{array}\right) \Delta^{m-k-1} \operatorname{div}^{l}\left(\varphi\left\langle D^{k+1} u, D^{k-l+1} e_{i}\right\rangle\right) \\
& \Delta^{m} \eta_{k, l}^{i}=(-1)^{k-l}\left(\begin{array}{c}
k \\
l
\end{array}\right) \Delta^{m-k-1} \operatorname{div}^{l+1}\left(\varphi\left\langle D^{k} u, D^{k-l+1} e_{i}\right\rangle\right)
\end{aligned}
$$

with the exception

$$
\eta_{0,0}^{i}:=0
$$

Note that on $B_{1 / 2}$, where $\varphi \equiv 1$, the right-hand sides are simply the terms from the Euler-Lagrange equation (4.4); hence (6.2) allows us to rewrite the Euler-Lagrange equation for $u$ as

$$
0=\Delta^{m}\left[f_{i}-\sum_{0 \leq k \leq l<m}\left(\omega_{k, l}^{i}+\eta_{k, l}^{i}\right)\right]=: \Delta^{m}\left[f_{i}-w_{i}\right]
$$

in the sense of distributions on $B_{1 / 2}$.

Lemma 6.1. The fundamental solution for $\Delta^{m}$ on $\mathbb{R}^{2 m}$ is $G(x):=c_{m} \ln |x|$. We have

$$
D^{k} G \in L^{2 m / k, \infty}\left(\mathbb{R}^{2 m}\right)
$$

for $k=1, \ldots, 2 m-1$, and $D^{2 m} G$ is a Calderon-Zygmund kernel.

Proof. These are straightforward computations.

Now we are going to estimate $D^{m} \omega_{k, l}^{i}$ and $D^{m} \eta_{k, l}^{i}$ in $L^{2, \infty}$, very closely to [23, Lemma 4.3]. Unless stated otherwise, all norms are to be taken with $\mathbb{R}^{2 m}$ as domain.

Case 1: $m+1 \leq 3 m-2 k+l-2<2 m$. We have

$$
\begin{aligned}
\omega_{k, l}^{i}(x) & =c \int_{\mathbf{R}^{2 m}} G(x-y) \Delta^{m-k-1} \operatorname{div}^{l}\left(\varphi\left\langle D^{k+1} u, D^{k-l+1} e_{i}\right\rangle\right)(y) d y \\
& =c \int_{\mathbf{R}^{2 m}}\left\langle\Delta^{m-k-1} D^{l} G(x-y),\left\langle D^{k+1} u, D^{k-l+1} e_{i}\right\rangle(y)\right\rangle \varphi(y) d y .
\end{aligned}
$$

This implies

$$
\left|D^{m} \omega_{k, l}^{i}(x)\right| \leq C \int_{\mathbf{R}^{2 m}}\left|D^{3 m-2 k+l-2} G\right|(x-y)\left|D^{k+1} u\right|\left|D^{k-l+1} e_{i}\right|(y) \varphi(y) d y
$$


which yields by the convolution inequality (2.6)

$$
\begin{aligned}
\left\|D^{m} \omega_{k, l}^{i}\right\|_{L^{2, \infty}} & \leq C\left\|\varphi \mid D^{k+1} u\right\| D^{k-l+1} e_{i}\|\|_{L^{2 m /(2 k-l+2), \infty}} \\
& \leq C\left\|D^{k+1} u\right\|_{L^{2 m /(k+1), \infty}(B)}\left\|D^{k-l+1} e_{i}\right\|_{L^{2 m /(k-l+1), \infty}(B)}
\end{aligned}
$$

Case 2: $3 m-2 k+l-2 \geq 2 m$. In this case we perform only $m$ partial integrations and find

$$
\begin{aligned}
& \left|D^{m} \omega_{k, l}^{i}(x)\right| \\
& \quad \leq C \int_{\mathbf{R}^{2 m}}\left|D^{2 m} G\right|(x-y)\left|D^{m-2 k+l-2}\left(\varphi\left\langle D^{k+1} u, D^{k-l+1} e_{i}\right\rangle\right)\right|(y) d y .
\end{aligned}
$$

Using again the Calderon-Zygmund estimates from (2.8), we find

$$
\begin{aligned}
\left\|D^{m} \omega_{k, l}^{i}\right\|_{L^{2, \infty}} & \leq C\left\|D^{m-2 k+l-2}\left(\varphi\left\langle D^{k+1} u, D^{k-l+1} e_{i}\right\rangle\right)\right\|_{L^{2, \infty}} \\
& \leq C\|\varphi\|_{C^{m}} \sum_{h=1}^{m-1} \sum_{j=1}^{m-h}\left\|D^{h} u\right\|_{L^{2(h+j) / h, \infty}(B)}\left\|D^{j} e_{i}\right\|_{L^{2(h+j) / j, \infty}(B)} \\
& \leq C \sum_{h=1}^{m-1} \sum_{j=1}^{m-h}\left\|D^{h} u\right\|_{L^{2 m / h, \infty}(B)}\left\|D^{j} e_{i}\right\|_{L^{2 m / j, \infty}(B)} .
\end{aligned}
$$

Case 3: $3 m-2 k+l-2=m$, that is $k=m-1$ and $l=0$. Here we have

$$
\omega_{k, l}^{i}(x)=\int_{\mathbf{R}^{2 m}} G(x-y) \varphi(y)\left\langle D^{m} u, D^{m} e_{i}\right\rangle(y) d y
$$

and hence

$$
\left|D^{m} \omega_{k, l}^{i}(x)\right| \leq C \int_{\mathbf{R}^{2 m}}\left|D^{m} G\right|(x-y) \varphi(y)\left|\left\langle D^{m} u, D^{m} e_{i}\right\rangle\right|(y) d y .
$$

To deal with the term $\left|\left\langle D^{m} u, D^{m} e_{i}\right\rangle\right|$, we introduce the orthogonal projections $\Pi(y): \mathbb{R}^{L} \rightarrow \mathrm{T}_{y} N$ and $\Pi^{\perp}(y): \mathbb{R}^{L} \rightarrow \mathrm{T}_{y}^{\perp} N$ and write

$$
\begin{aligned}
\left\langle D^{m} u, D^{m} e_{i}\right\rangle= & \left\langle(\Pi \circ u) D^{m} u, D^{m-1}\left(\left(\Pi^{\perp} \circ u\right) D e_{i}\right)\right\rangle \\
& +\left\langle(\Pi \circ u) D^{m} u, D^{m-1}\left((\Pi \circ u) D e_{i}\right)\right\rangle \\
& \left.+\left\langle\left(\Pi^{\perp} \circ u\right) D^{m} u, D^{m} e_{i}\right)\right\rangle \\
= & : I+I I+I I I .
\end{aligned}
$$


In order to estimate $I$, we calculate

$$
\begin{aligned}
I & =\sum_{k=0}^{m-1}\left(\begin{array}{c}
m-1 \\
k
\end{array}\right)\left\langle(\Pi \circ u) D^{m} u, D^{k}\left(\Pi^{\perp} \circ u\right) D^{m-k} e_{i}\right\rangle \\
& =\sum_{k=1}^{m-1}\left(\begin{array}{c}
m-1 \\
k
\end{array}\right)\left\langle(\Pi \circ u) D^{m} u, D^{k}\left(\Pi^{\perp} \circ u\right) D^{m-k} e_{i}\right\rangle
\end{aligned}
$$

where we used $\left\langle(\Pi \circ u) D^{m} u,\left(\Pi^{\perp} \circ u\right) D^{m} e_{i}\right\rangle=0$ in the last step. From the above identity, the chain rule and Young's inequality we deduce

$$
|I| \leq C\left|D^{m} u\right| \sum_{k=1}^{m-1} \sum_{j=1}^{k}\left|D^{j} u\right|^{k / j}\left|D^{m-k} e_{i}\right|
$$

Here we used $\left\|\Pi^{\perp}\right\|_{C^{m-1}} \leq C(N)$. Integrating the above inequality, we get

$$
\begin{aligned}
\|\varphi I\|_{L^{1}} & \leq C\left\|D^{m} u\right\|_{L^{2, \infty}(B)}\left\|\sum_{k=1}^{m-1} \sum_{j=1}^{k}\left|D^{j} u\right|^{k / j}\left|D^{m-k} e_{i}\right|\right\|_{L^{2,1}(B)} \\
& \leq C\left\|D^{m} u\right\|_{L^{2, \infty}(B)} \sum_{k=1}^{m-1} \sum_{j=1}^{k}\left\|D^{j} u\right\|_{L^{2 m / j, 2}(B)}^{k / j}\left\|D^{m-k} e_{i}\right\|_{L^{2 m /(m-k), 2}(B)} \\
& \leq C\left\|D^{m} u\right\|_{L^{2, \infty}(B)}\left\|D e_{i}\right\|_{W^{m-1,2}(B)} \sum_{1 \leq j \leq k<m}\left\|D^{j} u\right\|_{L^{2 m / j, 2}(B)}^{k / j}
\end{aligned}
$$

where we used the estimate

$$
\left\|\left|D^{j} u\right|^{k / j}\right\|_{L^{2 m / k, 2}}=\left\|D^{j} u\right\|_{L^{2 m / j, 2 k / j}}^{k / j} \leq C\left\|D^{j} u\right\|_{L^{2 m / j, 2}}^{k / j}
$$

in the second step and the Sobolev-Lorentz embedding (2.4) in the last inequality. For the estimate of the second term, we use the identity $(\Pi \circ u)$ $D e_{i}=\sum_{l=1}^{\operatorname{dim} N} A_{l i} e_{l}$, from which we conclude

$$
|I I| \leq C\left|D^{m} u\right| \sum_{j=1}^{m}\left|D^{j-1} A\right|\left|D^{m-j} e_{i}\right| .
$$

This implies by $(2.10)$

$$
\|\varphi I I\|_{L^{1}} \leq C\left\|D^{m} u\right\|_{L^{2, \infty}(B)}\left\|\sum _ { j = 1 } ^ { m } \left|D^{j-1} A\left\|D^{m-j} e_{i} \mid\right\|_{L^{2,1}(B)}\right.\right.
$$




$$
\begin{aligned}
\leq & C\left\|D^{m} u\right\|_{L^{2, \infty}(B)} \\
& \times\left(\left\|D^{m-1} A\right\|_{L^{2,1}(B)}+\sum_{j=1}^{m-1}\left\|D^{j-1} A\right\|_{L^{2 m / j, 2}}\left\|D^{m-j} e_{i}\right\|_{L^{2 m /(m-j), 2}}\right) \\
\leq & C\left\|D^{m} u\right\|_{L^{2, \infty}(B)}\left(\left\|D^{m-1} A\right\|_{L^{2,1}(B)}+\|A\|_{W^{m-1,2}(B)}\left\|D e_{i}\right\|_{W^{m-1,2}(B)}\right) .
\end{aligned}
$$

Here we have applied the Sobolev-Lorentz embedding theorem in the last step. Finally, to estimate the third term we use the equality $D^{m-1}\left(\left(\Pi^{\perp}\right.\right.$ 。 $u) D u)=0$ and Leibniz' rule to infer

$$
\begin{aligned}
\left|\left(\Pi^{\perp} \circ u\right) D^{m} u\right| & \leq C \sum_{k=1}^{m-1}\left|D^{k}\left(\Pi^{\perp} \circ u\right)\right|\left|D^{m-k} u\right| \\
& \leq C \sum_{k=1}^{m-1} \sum_{j=1}^{k}\left|D^{j} u\right|^{k / j}\left|D^{m-k} u\right|
\end{aligned}
$$

where we used Young's inequality in the last step. The last estimate yields

$$
|I I I| \leq C\left|D^{m} e_{i}\right| \sum_{k=1}^{m-1} \sum_{j=1}^{k}\left|D^{j} u\right|^{k / j}\left|D^{m-k} u\right|
$$

which implies, similarly to the derivation of (6.7),

$$
\|\varphi I I I\|_{L^{1}} \leq\left\|D^{m} e_{i}\right\|_{L^{2}(B)} \sum_{k=1}^{m-1} \sum_{j=1}^{k}\left\|D^{j} u\right\|_{L^{2 m / j, 2}(B)}^{k / j}\left\|D^{m-k} u\right\|_{L^{2 m /(m-k), \infty}(B)} .
$$

Now we use $D^{m} G \in L^{2, \infty}$ combined with the convolution estimate (2.7) in (6.6). Putting Equations (6.7) to (6.9) together, we conclude

$$
\begin{aligned}
& \left\|D^{m} \omega_{k, l}^{i}\right\|_{L^{2, \infty}} \leq\left\|\varphi\left\langle D^{m} u, D^{m} e_{i}\right\rangle\right\|_{L^{1}} \\
& \leq C \sum_{i=1}^{m}\left\|D^{i} u\right\|_{L^{2 m / i, \infty}(B)}\left\|D e_{i}\right\|_{W^{m-1,2}(B)} \sum_{1 \leq j \leq k<m}\left\|D^{j} u\right\|_{L^{2 m / j, 2}(B)}^{k / j} \\
& \quad+C\left\|D^{m} u\right\|_{L^{2, \infty}(B)}\left(\left\|D^{m-1} A\right\|_{L^{2,1}(B)}+\|A\|_{W^{m-1,2}(B)}\left\|D e_{i}\right\|_{W^{m-1,2}(B)}\right) .
\end{aligned}
$$


The estimates for the $\eta_{k, l}^{i}$ are completely analogous to Cases 1 and 2 above. We have

$$
\begin{aligned}
\left\|D^{m} \eta_{k, l}^{i}\right\|_{L^{2, \infty}} & \leq C\left\|\varphi \mid D^{k} u\right\| D^{k-l+1} e_{i}\|\|_{L^{2 m /(2 k-l+1), \infty}} \\
& \leq C\left\|D^{k} u\right\|_{L^{2 m / k, \infty}(B)}\left\|D^{k-l+1} e_{i}\right\|_{L^{2 m /(k-l+1), \infty}(B)}
\end{aligned}
$$

in the case $3 m-2 k+l-1<2 m$, and

$$
\left\|D^{m} \eta_{k, l}^{i}\right\|_{L^{2, \infty}} \leq \sum_{h=1}^{m-1} \sum_{j=1}^{m-h}\left\|D^{h} u\right\|_{L^{2 m / h, \infty}(B)}\left\|D^{j} e_{i}\right\|_{L^{2 m / j, \infty}(B)}
$$

for $3 m-2 k+l-1 \geq 2 m$. Recall that here we need not consider $k=0$. We abbreviate

$$
\begin{aligned}
R_{\infty} & :=\sum_{k=1}^{m}\left\|D^{k} u\right\|_{L^{2 m / k, \infty}(B)} \\
S & :=\sum_{k=1}^{m}\left(\left\|D^{k} u\right\|_{L^{2 m / k}(B)}+\left\|D^{k-1} A\right\|_{L^{2 m / k}(B)}\right)^{m / k}, \\
S_{p} & :=\sum_{k=1}^{m}\left(\left\|D^{k} u\right\|_{L^{2 m / k, p}(B)}+\left\|D^{k-1} A\right\|_{L^{2 m / k, p}(B)}\right)^{m / k} .
\end{aligned}
$$

Lemma 5.3 yields the estimate $\left\|D^{k} e_{i}\right\|_{L^{2 m / k, \infty}} \leq C S_{\infty}^{k / m}$. Thus, the righthand sides of $(6.4),(6.5),(6.11)$ and $(6.12)$ can all be estimated by $C S_{\infty}^{1 / m}$ $R_{\infty}$, since we can assume $S_{\infty}<1$ by (6.1). We combine this with $(6.10)$ to obtain

$$
\begin{aligned}
\left\|D^{m} w_{i}\right\|_{L^{2, \infty}} \leq & C S_{\infty}^{1 / m} R_{\infty}+C S_{2}^{1 / m}\left\|D e_{i}\right\|_{W^{m-1,2}(B)} R_{\infty} \\
& +C\left(\left\|D^{m-1} A\right\|_{L^{2,1}(B)}+\|A\|_{W^{m-1,2}(B)}\left\|D e_{i}\right\|_{W^{m-1,2}(B)}\right) R_{\infty}
\end{aligned}
$$

Now we apply (6.1) and Lemma 5.3 to find, assuming $\varepsilon_{0}<1$,

$$
\begin{aligned}
\left\|D^{m-1} A\right\|_{L^{2,1}}+\|A\|_{W^{m-1,2}}+ & \left\|D e_{i}\right\|_{W^{m-1,2}} \leq C \varepsilon_{0} \\
S+S_{2}+S_{\infty} & \leq C \varepsilon_{0}
\end{aligned}
$$

where in the last estimate we have used the Sobolev-Lorentz embedding. We use this in (6.13) to find

$$
\left\|D^{m} w_{i}\right\|_{L^{2, \infty}} \leq C \varepsilon_{0}^{1 / m} R_{\infty}
$$


and, using the Sobolev-Lorentz embedding (2.3),

$$
\sum_{k=1}^{m}\left\|D^{k} w_{i}\right\|_{L^{2 m / k, \infty}\left(\mathbf{R}^{2 m}\right)} \leq C \varepsilon_{0}^{1 / m} R_{\infty} .
$$

Now we are going to exploit (6.3), i.e., the fact that $f_{i}-w_{i}$ is a polyharmonic function.

Lemma 6.2 (Growth estimate for polyharmonic functions). Suppose that $v \in C^{\infty}\left(B^{n}, \mathbb{R}^{K}\right)$, where $n \geq 2 m$, is a polyharmonic function of order $m$, that is $\Delta^{m} v=0$ in $B^{n}$. Then there is a constant $C$ depending only on $m$ and $n$, such that

$$
\|v\|_{L^{\infty}\left(B_{1 / 2}\right)} \leq C\|v\|_{L^{1}(B)}
$$

and furthermore, for all $\theta \in\left(0, \frac{1}{2}\right)$ we have

$$
\sum_{j=1}^{m}\left\|D^{j} v\right\|_{L^{2 m / j, \infty}\left(B_{\theta}\right)} \leq C \theta \sum_{j=1}^{m}\left\|D^{j} v\right\|_{L^{2 m / j, \infty}(B)} .
$$

Proof. For the proof we employ a mean value identity for polyharmonic functions, see [3], which reads as follows. For any choice of radii $0<r_{1}<$ $r_{2}<\cdots<r_{m}$, there are coefficients $\lambda_{i} \in \mathbb{R}$ depending only on $m$ and the $r_{i}$, such that every polyharmonic function $h \in C^{2 m}\left(B_{r_{m}}(x), \mathbb{R}^{K}\right)$ of order $m$ satisfies

$$
h(x)=\sum_{i=1}^{m} \lambda_{i} f_{B_{r_{i}}(x)} h(y) d y .
$$

We apply this identity with $x \in B_{1 / 2}$ and radii $r_{i} \in\left[\frac{1}{4}, \frac{1}{2}\right]$ to infer

$$
\|h\|_{L^{\infty}\left(B_{1 / 2}\right)} \leq C(m, n)\|h\|_{L^{1}(B)} .
$$

This proves (6.18) if we choose $h=v$. Next we choose $h=D^{j} v$ for $j=$ $1, \ldots, m$, which are again polyharmonic functions, and estimate

$$
\begin{aligned}
\left\|D^{j} v\right\|_{L^{2 m / j, \infty}\left(B_{\theta}\right)} & \leq C \theta^{j}\left\|D^{j} v\right\|_{L^{\infty}\left(B_{1 / 2}\right)} \leq C \theta^{j}\left\|D^{j} v\right\|_{L^{1}(B)} \\
& \leq C \theta^{j}\left\|D^{j} v\right\|_{L^{2 m / j, \infty}(B)}
\end{aligned}
$$

for all $j=1, \ldots, m$ and $\theta<\frac{1}{2}$, where the constant $C$ depends only on $m$ and $n$. 
Returning to the proof of Proposition 6.1, we apply the last lemma to $f_{i}-w_{i}$. Since that function is polyharmonic on $B_{1 / 2}$, the lemma can be applied for $\theta \in\left(0, \frac{1}{4}\right)$ to find, using $(6.17)$ twice,

$$
\begin{aligned}
\sum_{k=1}^{m}\left\|D^{k} f_{i}\right\|_{L^{2 m / k, \infty}\left(B_{\theta}\right)} & \leq \sum_{k=1}^{m}\left(\left\|D^{k}\left(f_{i}-w_{i}\right)\right\|_{L^{2 m / k, \infty}\left(B_{\theta}\right)}+\left\|D^{k} w_{i}\right\|_{L^{2 m / k, \infty}\left(\mathbf{R}^{2 m}\right)}\right) \\
& \leq C \theta \sum_{k=1}^{m}\left\|D^{k}\left(f_{i}-w_{i}\right)\right\|_{L^{2 m / k, \infty}\left(B_{1 / 2}\right)}+C \varepsilon_{0}^{1 / m} R_{\infty} \\
& \leq C \theta \sum_{k=1}^{m}\left\|D^{k} f_{i}\right\|_{L^{2 m / k, \infty}\left(B_{1 / 2}\right)}+C \varepsilon_{0}^{1 / m} R_{\infty} .
\end{aligned}
$$

On the other hand, using Lemma 3.1 and the arguments from (6.5), as well as $(2.5)$ and $\varepsilon_{0} \leq 1$, we estimate

$$
\begin{aligned}
\sum_{k=1}^{m}\left\|D^{k} f_{i}\right\|_{L^{2 m / k, \infty}\left(\mathbf{R}^{2 m}\right) \leq} & C \sum_{k=1}^{m}\left\|D^{k-1}\left\langle D(\varphi(u-\bar{u})), e_{i}\right\rangle\right\|_{L^{2 m / k, \infty}\left(\mathbf{R}^{2 m}\right)} \\
\leq & C R_{\infty} S_{\infty}^{1 / m}+C\|u-\bar{u}\|_{L^{2 m, \infty}(B)} \\
& \times \sum_{j=1}^{m-1}\left\|D^{j} e_{i}\right\|_{L^{2 m / j, \infty}(B)}+\sum_{k=1}^{m}\left\|D^{k} u\right\|_{L^{2 m, \infty}(B)} \\
\leq & C R_{\infty} .
\end{aligned}
$$

Combining the last two estimates, we have proven

$$
\sum_{k=1}^{m}\left\|D^{k} f_{i}\right\|_{L^{2 m / k, \infty}\left(B_{\theta}\right)} \leq C\left(\theta+\varepsilon_{0}^{1 / m}\right) R_{\infty} .
$$

Now we turn our attention to estimating $g_{i}$. Again from Lemma 3.1 we know

$$
\begin{aligned}
\sum_{k=2}^{m}\left\|D^{k} g_{i}\right\|_{L^{2 m / k, \infty}\left(\mathbf{R}^{2 m}\right)} \leq & C \sum_{k=2}^{m}\left\|D^{k-2}\left[d(\varphi(u-\bar{u})) \wedge d e_{i}\right]\right\|_{L^{2 m / k, \infty}\left(\mathbf{R}^{2 m}\right)} \\
\leq & C \sum_{h=1}^{m-1} \sum_{j=1}^{m-h}\left\|D^{h} u\right\|_{L^{2 m / h, \infty}(B)}\left\|D^{j} e_{i}\right\|_{L^{2 m / j, \infty}(B)} \\
& +C\|u-\bar{u}\|_{L^{2 m, \infty}(B)} \sum_{j=1}^{m-1}\left\|D^{j} e_{i}\right\|_{L^{2 m / j, \infty}(B)}
\end{aligned}
$$




$$
\begin{aligned}
& \leq C \sum_{h=1}^{m-1} \sum_{j=1}^{m-h}\left\|D^{h} u\right\|_{L^{2 m / h, \infty}(B)}\left\|D^{j} e_{i}\right\|_{L^{2 m / j, \infty}(B)} \\
& \leq C S_{\infty}^{1 / m} R_{\infty} \\
& \leq C \varepsilon_{0}^{1 / m} R_{\infty} .
\end{aligned}
$$

By the Sobolev-Lorentz embedding (2.3), we even know

$$
\sum_{k=1}^{m}\left\|D^{k} g_{i}\right\|_{L^{2 m / k, \infty}\left(\mathbf{R}^{2 m}\right)} \leq C \varepsilon_{0}^{1 / m} R_{\infty}
$$

From (6.20) and (6.21), we conclude

$$
\sum_{k=1}^{m}\left\|D^{k-1}\left\langle D u, e_{i}\right\rangle\right\|_{L^{2 m / k, \infty}\left(B_{\theta}\right)} \leq C\left(\theta+\varepsilon_{0}^{1 / m}\right) R_{\infty}
$$

which by Leibniz' rule for all $1 \leq k \leq m$ implies

$$
\begin{aligned}
\left\|D^{k} u\right\|_{L^{2 m / k, \infty}\left(B_{\theta}\right)}= & \left\|D^{k-1}\left(\sum_{i}\left\langle D u, e_{i}\right\rangle e_{i}\right)\right\|_{L^{2 m / k, \infty}\left(B_{\theta}\right)} \\
\leq & \left\|D^{k-1}\left\langle D u, e_{i}\right\rangle\right\|_{L^{2 m / k, \infty}\left(B_{\theta}\right)} \\
& +C \sum_{i} \sum_{\ell=1}^{k-1}\left\|D^{\ell-1}\left\langle D u, e_{i}\right\rangle\right\|_{L^{2 m / \ell, \infty}\left(B_{\theta}\right)} \\
& \times\left\|D^{k-\ell} e_{i}\right\|_{L^{2 m /(k-\ell), \infty}\left(B_{\theta}\right)} \\
\leq & C\left(\theta+\varepsilon_{0}^{1 / m}\right) R_{\infty}\left(1+S_{\infty}^{1 / m}\right) .
\end{aligned}
$$

This is the assertion of Proposition 6.1 if we choose $\theta$ and $\varepsilon_{0}$ small enough.

\section{Proof of the regularity theorem}

By the absolute continuity of the integral, we find an $r_{0}>0$ such that

$$
R\left(x_{0}, r\right):=\sum_{k=1}^{m}\left\|D^{k} u\right\|_{L^{2 m / k}\left(B_{r}\left(x_{0}\right)\right)} \leq \varepsilon_{0} \quad \text { for all } x_{0} \in \Omega \text { and } r \leq r_{0}
$$


Therefore and because of the scaling invariance of $R\left(x_{0}, r\right)$, we can use and iterate Proposition 6.1 on all balls $B_{r}\left(x_{0}\right)$ with $r \leq r_{0}$ to infer

$$
\sum_{k=1}^{m}\left\|D^{k} u\right\|_{L^{2 m / k, \infty}\left(B_{r}\left(x_{0}\right)\right)}<C r^{\alpha} \quad \text { for all } x_{0} \in \Omega \text { and } r \leq r_{0}
$$

for some $\alpha \in(0,1)$. By $(2.2)$, this implies

$$
r^{p-2 m} \int_{B_{r}\left(x_{0}\right)}|D u|^{p} d x \leq C_{p}\|D u\|_{L^{2 m, \infty}\left(B_{r}\left(x_{0}\right)\right)}^{p} \leq C r^{p \alpha}
$$

for all $1 \leq p<2 m, x_{0} \in \Omega$, and $r \leq r_{0}$. Then $u \in C^{0, \alpha}(\Omega)$. The higher regularity can now be derived from the following proposition, which is proved by techniques that have been sketched for the biharmonic map case in [4].

Proposition 7.1. For $n \geq 2 m$, any (extrinsically or intrinsically) $m$-polyharmonic map $u \in C^{0, \alpha} \cap W^{m, 2}\left(B^{n}, N\right)$ satisfies $u \in C^{\infty}\left(B^{n}, N\right)$.

Remark 7.1. The proof shows that the proposition holds more generally for maps $u \in C^{0, \alpha} \cap W^{m, 2}\left(B^{n}, \mathbb{R}^{K}\right)$ that satisfy, in the distributional sense, a differential equation of the form

$\Delta^{m} u=\sum_{k=0}^{m-1} \operatorname{div}^{k} g_{k}, \quad$ where $\quad\left|g_{k}\right| \leq C \sum_{l=1}^{m}\left|D^{l} u\right|^{(2 m-k) / l} \quad$ for $0 \leq k \leq m-1$.

Extrinsically polyharmonic maps satisfy a differential equation of this form, as can be checked by performing integration by parts in the first sum of the Euler equation (4.2) and applying Young's inequality on the $k$-linear forms $g_{k}$. In Section 8, we will show that intrinsically polyharmonic maps satisfy an equation of the type (7.2) as well.

Before proceeding to the proof of the proposition, we give the following

Lemma 7.1. Assume that for $n \geq 2 m$, the map $u \in W^{m, 2}\left(B^{n}, \mathbb{R}^{K}\right)$ satisfies

$$
\Delta^{m} u=\sum_{k=0}^{m-1} \operatorname{div}^{k} g_{k} \quad \text { on } B^{n}
$$


in the distributional sense, where the $k$-linear forms $g_{k}$ satisfy

$$
\left(r^{2 m-n} \int_{B_{r}(x)}\left|g_{k}\right|^{2 m /(2 m-k)} d x\right)^{(2 m-k) / 2 m} \leq K r^{\gamma} \quad \text { for any ball } B_{r}(x) \subset B
$$

for some $0<\gamma<m$ and a constant $K$. Then for all $x \in B$ and $0<\rho<$ $\frac{1}{3}(1-|x|)$, we have

$$
\begin{aligned}
& \left(\rho^{2 l-n} \int_{B_{\rho}(x)}\left|D^{l} u\right|^{2} d x\right)^{1 / 2} \\
& \quad \leq C \rho^{\tilde{\gamma}}\left[1+(1-|x|)^{l-\gamma-\frac{n}{2}}\left\|D^{l} u\right\|_{L^{2}}\right] \quad \text { for } \gamma<l \leq m
\end{aligned}
$$

If the assumptions are satisfied for $m<\gamma<m+1$, then there holds

$$
\begin{aligned}
& \left(\rho^{2 m-n} \int_{B_{\rho}(x)}\left|D^{m} u-\left(D^{m} u\right)_{x, \rho}\right|^{2} d x\right)^{1 / 2} \\
& \quad \leq C \rho^{\tilde{\gamma}}\left[1+(1-|x|)^{m-\gamma-(n / 2)}\left\|D^{m} u\right\|_{L^{2}}\right]
\end{aligned}
$$

for $x \in B$ and $0<\rho<\frac{1}{3}(1-|x|)$. These estimates hold for any $\tilde{\gamma}<\gamma$, and in the supercritical case $n>2 m$ even for $\tilde{\gamma}=\gamma$. We employed the notation $\left(D^{m} u\right)_{x, \rho}:=f_{B_{\rho}(x)} D^{m} u$. The constants $C$ in the above estimates depend only on $m, n$ and $K$, and on $\tilde{\gamma}-\gamma$ in the critical case.

Proof. Fix $x \in B$ and $0<r \leq \frac{1}{3}(1-|x|)$ and let $v \in C^{\infty}\left(B_{r}(x), \mathbb{R}^{K}\right)$ be the polyharmonic function with $w:=u-v \in W_{0}^{m, 2}\left(B_{r}(x), \mathbb{R}^{K}\right)$. In the proof of $L^{\infty}$-estimates for $w$, we will use the Green function $G_{m, n}: B \rightarrow \mathbb{R}$ for $\Delta^{m}$ on the $n$-dimensional unit ball $B$ (with Dirichlet boundary data for the solution and its first $m-1$ normal derivatives). This Green function is known explicitly, and Grunau and Sweers have given the exact growth in [10].

We start by approximating $w \in W_{0}^{m, 2}\left(B_{r}(x), \mathbb{R}^{K}\right)$ by smooth functions $w_{i} \in C_{\mathrm{cpt}}^{\infty}\left(B_{r}(x), \mathbb{R}^{K}\right)$ in the sense $w_{i} \rightarrow w$ in $W^{m, 2}\left(B_{r}(x), \mathbb{R}^{K}\right)$-norm as $i \rightarrow$ $\infty$. The approximating functions satisfy

$$
\left|w_{i}(z)\right|=r^{m-n}\left|\int_{B_{r}(x)}\left\langle D^{m} G_{m, n}\left(\frac{z-x}{r}, \frac{y-x}{r}\right), D^{m} w_{i}(y)\right\rangle d y\right| .
$$

We cannot pass to the limit $i \rightarrow \infty$ on the right-hand side pointwise in $z$, but we are allowed to read both sides as functions of $z$ and pass to the limit 
in the $L^{1}$ sense. Hence we have

$$
|w(z)|=r^{m-n}\left|\int_{B_{r}(x)}\left\langle D^{m} G_{m, n}\left(\frac{z-x}{r}, \frac{y-x}{r}\right), D^{m} w(y)\right\rangle d y\right|
$$

for almost all $z \in B_{r}(x)$. Derivatives of $G_{m, n}$ are to be understood with respect to the second variable. We would like to test (7.3) with the above rescaled Green function to find

$$
|w(z)| \leq \sum_{k=0}^{m-1} r^{2 m-n-k}\left|\int_{B_{r}(x)}\left\langle D^{k} G_{m, n}\left(\frac{z-x}{r}, \frac{y-x}{r}\right), g_{k}(y)\right\rangle d y\right|
$$

and in fact we may do so for almost all $z \in B_{r}(x)$ by the same reasoning as above, this time approximating $G_{m, n}(\cdot, y)$ in $W^{m, 1}(B)$ by smooth functions with compact support, uniformly in $y \in B$. From [10, Propositions 2.3 and 2.4 ], we know that

$$
\left|D^{k} G_{m, n}(X, Y)\right| \leq C|X-Y|^{2 m-n-k}
$$

for all $X, Y \in B$ whenever $k>2 m-n$. Therefore, in the supercritical case $n>2 m$,

$$
|w(z)| \leq C \sum_{k=0}^{m-1} \int_{B_{r}(x)}|z-y|^{2 m-n-k}\left|g_{k}(y)\right| d y .
$$

Integration by parts gives

$$
\begin{aligned}
& \int_{B_{r}(x)}|z-y|^{2 m-n-k}\left|g_{k}(y)\right| d y \\
& \leq \int_{0}^{2 r} \rho^{2 m-n-k} \int_{S_{\rho}(z)}\left|g_{k}\right| d \mathcal{H}^{n-1} d \rho \\
& \leq(2 r)^{2 m-n-k} \int_{B_{2 r}(z)}\left|g_{k}(y)\right| d y+(n+k-2 m) \\
& \quad \times \int_{0}^{2 r} \rho^{2 m-n-k-1} \int_{B_{\rho}(z)}\left|g_{k}(y)\right| d y d \rho \\
& \leq C r^{\gamma}+C \int_{0}^{2 r} \rho^{\gamma-1} d \rho \leq C r^{\gamma}
\end{aligned}
$$

by the assumptions on $g_{k}$. Combining the last two estimates, we have established

$$
\|w\|_{L^{\infty}\left(B_{r}(x)\right)} \leq C r^{\gamma}
$$


whenever $n>2 m$. In the critical case $n=2 m$, we only have

$$
\left|G_{m, n}(X, Y)\right| \leq C|\log | X-Y||
$$

while for the derivatives the estimates hold as cited above. This allows only a modified version of the calculation above, to the effect that

$$
\|w\|_{L^{\infty}\left(B_{r}(x)\right)} \leq C r^{\gamma}|\log r|
$$

In both cases, we have

$$
\|w\|_{L^{\infty}\left(B_{r}(x)\right)} \leq C r^{\tilde{\gamma}}
$$

for all $\tilde{\gamma}<\gamma$ resp. $\tilde{\gamma} \leq \gamma$, as in the statement.

By Nirenberg interpolation, this implies $\left|D^{k} w\right| \in L^{2 m / k}\left(B_{r}(x)\right)$ for all $0<k \leq m-1$,

$$
\begin{aligned}
& \left(r^{2 m-n} \int_{B_{r}(x)}\left|D^{k} w\right|^{2 m / k} d x\right)^{k / 2 m} \\
& \leq C r^{\tilde{\gamma}\left(1-\frac{k}{m}\right)}\left(r^{2 m-n} \int_{B_{r}(x)}\left|D^{m} w\right|^{2} d x\right)^{k / 2 m}+C r^{\tilde{\gamma}} \\
& \leq \frac{1}{2 m} r^{2 m-n-\tilde{\gamma}} \int_{B_{r}(x)}\left|D^{m} w\right|^{2} d x+C r^{\tilde{\gamma}}
\end{aligned}
$$

where we applied Young's inequality with the exponents $\frac{2 m}{2 m-k}$ and $\frac{2 m}{k}$ in the second step. Now we test Equation (7.3) with $w:=u-v \in W_{0}^{m, 2}\left(B_{r}(x), \mathbb{R}^{K}\right)$ and get, because $\Delta^{m} v=0$,

$$
\begin{aligned}
r^{2 m-n} & \int_{B_{r}(x)}\left|D^{m} w\right|^{2} d x=r^{2 m-n} \int_{B_{r}(x)}\left\langle D^{m} u, D^{m} w\right\rangle d x \\
= & r^{2 m-n} \sum_{k=0}^{m-1} \int_{B_{r}(x)}\left\langle g_{k}, D^{k} w\right\rangle d x \\
\leq & \left.\sum_{k=0}^{m-1}\left(r^{2 m-n} \int_{B_{r}(x)}\left|D^{k} w\right|^{2 m / k} d x\right)^{k / 2 m}\right)^{(2 m-k) / 2 m} .
\end{aligned}
$$


Using estimates (7.6) in the case $k=0$ and (7.7) in the cases $1 \leq k \leq m-1$, combined with the assumptions on $g_{k}$, we arrive at

$$
r^{2 m-n} \int_{B_{r}(x)}\left|D^{m} w\right|^{2} d x \leq \frac{1}{2} r^{2 m-n} \int_{B_{r}(x)}\left|D^{m} w\right|^{2} d x+C r^{2 \tilde{\gamma}} .
$$

Absorbing the second integral, we conclude

$$
r^{2 m-n} \int_{B_{r}(x)}\left|D^{m} w\right|^{2} d x \leq C r^{2 \tilde{\gamma}}
$$

which implies by Nirenberg interpolation, cf. (7.7),

$$
\sum_{k=1}^{m}\left(r^{2 m-n} \int_{B_{r}(x)}\left|D^{k} w\right|^{2 m / k} d x\right)^{k / 2 m} \leq C r^{\tilde{\gamma}}
$$

Furthermore, by the $L^{\infty}$-estimates (6.18) of Lemma 6.2 we know for $1 \leq l \leq$ $m$, since $D^{l} v$ are polyharmonic functions,

$$
\int_{B_{\rho}(x)}\left|D^{l} v\right|^{2} d x \leq C\left(\frac{\rho}{r}\right)^{n} \int_{B_{r}(x)}\left|D^{l} v\right|^{2} d x
$$

for all $0<\rho<\frac{r}{2}$. Letting $\Psi_{l}(r):=\int_{B_{r}(x)}\left|D^{l} u\right|^{2} d x$, estimates (7.8) and (7.9) yield

$$
\Psi_{l}(\rho) \leq C\left(\frac{\rho}{r}\right)^{n} \int_{B_{r}(x)}\left|D^{l} v\right|^{2} d x+C r^{n-2 l+2 \tilde{\gamma}} \leq C\left(\frac{\rho}{r}\right)^{n} \Psi_{l}(r)+C r^{n-2 l+2 \tilde{\gamma}}
$$

for $0<\rho<\frac{r}{2}$ and $1 \leq l \leq m$. Note that for $\frac{r}{2} \leq \rho \leq r$, the above inequality holds trivially with $C=2^{n}$. Thus, for $l>\gamma$, a standard iteration argument [8, p.86] implies

$$
\Psi_{l}(\rho) \leq C\left(\frac{\rho}{r}\right)^{n-2 l+2 \tilde{\gamma}} \Psi_{l}(r)+C \rho^{n-2 l+2 \tilde{\gamma}}
$$

for all $\rho<r \leq \frac{1}{3}(1-|x|)$. This implies the claim (7.4). In order to prove the second claim, we need a Caccioppoli-type inequality for the polyharmonic function $v$. Applying the Poincaré inequality repeatedly, one checks

$$
r^{2 j-n} \int_{B_{r}(x)}\left|D^{j}(v-P)\right|^{2} d x \leq C r^{2 m-n} \int_{B_{r}(x)}\left|D^{m} v-\left(D^{m} v\right)_{x, r}\right|^{2} d x
$$

for an appropriate polynomial $P$ of degree $m$ and all $1 \leq j<m$. We choose a cut-off function $\eta \in C_{\mathrm{cpt}}^{\infty}\left(B_{r}(x),[0,1]\right)$ with $\eta \equiv 1$ on $B_{r / 2}(x)$ and $\left\|D^{j} \eta\right\|_{L^{\infty}} \leq$ 
$C r^{-j}$ for $0 \leq j \leq m$. This implies $\left\|D^{j} \eta^{2 m+2}\right\|_{L^{\infty}} \leq C r^{-j} \eta^{2 m-j+2}$ for all $0 \leq$ $j \leq m$. Testing $\Delta^{m} D v=0$ with $\eta^{2 m+2} D(v-P)$, we infer

$$
\int \eta^{2 m+2}\left|D^{m+1} v\right|^{2} d x \leq C \sum_{j=1}^{m} r^{j-m-1} \int \eta^{m+1+j}\left|D^{m+1} v\right|\left|D^{j}(v-P)\right| d x .
$$

Applying the Cauchy-Schwarz inequality, we infer

$$
\begin{aligned}
\int_{B_{r / 2}(x)}\left|D^{m+1} v\right|^{2} d x & \leq \sum_{j=1}^{m} r^{2 j-2 m-2} \int_{B_{r}(x)}\left|D^{j}(v-P)\right|^{2} d x \\
& \leq \frac{C}{r^{2}} \int_{B_{r}(x)}\left|D^{m} v-\left(D^{m} v\right)_{x, r}\right|^{2} d x
\end{aligned}
$$

by (7.10). Since $D^{m+1} v$ is a polyharmonic function, the estimate (7.9) also holds for $l=m+1$, which implies

$$
\begin{aligned}
\int_{B_{\rho}(x)}\left|D^{m} v-\left(D^{m} v\right)_{x, \rho}\right|^{2} d x & \leq C \rho^{2} \int_{B_{\rho}(x)}\left|D^{m+1} v\right|^{2} d x \\
& \leq C \frac{\rho^{n+2}}{r^{n}} \int_{B_{r / 2}(x)}\left|D^{m+1} v\right|^{2} d x \\
& \leq C\left(\frac{\rho}{r}\right)^{n+2} \int_{B_{r}(x)}\left|D^{m} v-\left(D^{m} v\right)_{x, r}\right|^{2} d x
\end{aligned}
$$

for all $\rho<\frac{r}{4}$, where we applied the Caccioppoli inequality (7.11) in the last step. Combining (7.8) and (7.12), we arrive at

$$
\begin{aligned}
\Phi(\rho) & :=\int_{B_{\rho}(x)}\left|D^{m} u-\left(D^{m} u\right)_{x, \rho}\right|^{2} d x \\
& \leq C\left(\frac{\rho}{r}\right)^{n+2} \int_{B_{r}(x)}\left|D^{m} v-\left(D^{m} v\right)_{x, r}\right|^{2} d x+C r^{n-2 m+2 \tilde{\gamma}} \\
& \leq C\left(\frac{\rho}{r}\right)^{n+2} \Phi(r)+C r^{n-2 m+2 \tilde{\gamma}}
\end{aligned}
$$

where we applied estimate (7.8) twice. Since $\tilde{\gamma}<m+1$, we can iterate this estimate as above with the result

$$
\Phi(\rho) \leq C\left(\frac{\rho}{r}\right)^{n-2 m+2 \tilde{\gamma}} \Phi(r)+C \rho^{n-2 m+2 \tilde{\gamma}}
$$

for all $0<\rho<r \leq \frac{1}{3}(1-|x|)$. This implies the claim (7.5). 
Proof of the Proposition 6.1. First, we want to prove that the Hölder continuity of $u$ implies Morrey space estimates for $D^{m} u$. For this, we fix $0<R<1$ and $x \in B_{R}$. For $0<r<1-|x|$, we let

$$
\Phi(r):=\int_{B_{r}(x)}\left|D^{m} u\right|^{2} d x .
$$

From the Hölder continuity of $u$, we know

$$
\|u-\bar{u}\|_{L^{\infty}\left(B_{r}(x)\right)} \leq C r^{\alpha}[u]_{\alpha}
$$

where $[u]_{\alpha}$ denotes the Hölder seminorm of $u$ and $\bar{u}:=f_{B_{r}(x)} u d x$. Moreover, using Nirenberg interpolation [15], we know

$$
\begin{aligned}
& r^{2 m-n} \int_{B_{r}(x)}\left|D^{j} u\right|^{2 m / j} d x \\
& \leq C\|u-\bar{u}\|_{L^{\infty}\left(B_{r}(x)\right)}^{2(m-j) / j} r^{2 m-n} \int_{B_{r}(x)}\left|D^{m} u\right|^{2} d x+C\|u-\bar{u}\|_{L^{\infty}\left(B_{r}(x)\right)}^{2 m / j} \\
& \leq C\left(r^{\alpha}[u]_{\alpha}\right)^{2 / m} r^{2 m-n} \Phi(r)+C r^{2 \alpha}[u]_{\alpha}^{2}
\end{aligned}
$$

for $1 \leq j \leq m-1$. Here, we used $\|u\|_{L^{\infty}} \leq C(N)<\infty$ in the last step together with $r<1$ and $j \leq m-1$. Let $\kappa \in(0,1)$ be a constant to be chosen later. For $2 \leq p<\frac{2 m}{j}$, we have a similar estimate

$$
\begin{aligned}
r^{p j-n} \int_{B_{r}(x)}\left|D^{j} u\right|^{p} d x \leq & C\|u-\bar{u}\|_{L^{\infty}\left(B_{r}(x)\right)}^{(m-j) p / m}\left[r^{2 m-n} \Phi(r)\right]^{p j / 2 m} \\
& +C\|u-\bar{u}\|_{L^{\infty}\left(B_{r}(x)\right)}^{p} \\
\leq & C\|u-\bar{u}\|_{L^{\infty}\left(B_{r}(x)\right)}^{(2 m-j p) / m}\left[r^{2 m-n} \Phi(r)\right]^{p j / 2 m} \\
& +C\|u-\bar{u}\|_{L^{\infty}\left(B_{r}(x)\right)}^{2} \\
\leq & \kappa r^{2 m-n} \Phi(r)+C_{\kappa} r^{2 \alpha}[u]_{\alpha}^{2}
\end{aligned}
$$

where we used $p \geq 2$ in the second step and in the last step, we applied Young's inequality with exponents $\frac{2 m}{p j}$ and $\frac{2 m}{2 m-j p}$ and used (7.14). Here, the constant $C_{\kappa}$ depends on $\kappa, m, p$ and $n$. We choose a cut-off function $\eta \in$ $C_{\mathrm{cpt}}^{\infty}\left(B_{r}(x),[0,1]\right)$ satisfying $\eta \equiv 1$ on $B_{r / 2}(x)$ and $\left\|D^{k} \eta\right\|_{L^{\infty}} \leq C r^{-k}$ for all $0 \leq k \leq m$. The polyharmonic map $u$ satisfies an equation of the form (7.2). 
Testing this equation with $V:=\eta^{2 m}(u-\bar{u})$, we estimate

$$
\begin{aligned}
\int \eta^{2 m}\left|D^{m} u\right|^{2} d x & =\int \eta^{2 m}\left\langle D^{m} u, D^{m}(u-\bar{u})\right\rangle d x \\
\leq & C \sum_{j=0}^{m-1} \int\left|D^{m} u \| D^{m-j} \eta^{2 m}\right|\left|D^{j}(u-\bar{u})\right| d x \\
& +C \sum_{k=0}^{m-1} \sum_{j=0}^{k} \int\left|g_{k}\right|\left|D^{k-j} \eta^{2 m}\right|\left|D^{j}(u-\bar{u})\right| d x \\
= & : I+\sum_{0 \leq j \leq k<m} I_{j k} .
\end{aligned}
$$

Using $\left\|D^{m-j} \eta^{2 m}\right\|_{L^{\infty}} \leq C r^{j-m} \eta^{m+j}$, Young's inequality, and (7.14), we get

$$
\begin{aligned}
I & \leq \frac{1}{4} \int \eta^{2 m}\left|D^{m} u\right|^{2} d x+C \sum_{j=1}^{m-1} r^{2 j-2 m} \int \eta^{2 j}\left|D^{j} u\right|^{2} d x+C r^{n-2 m+2 \alpha}[u]_{\alpha}^{2} \\
& \leq \frac{1}{4} \int \eta^{2 m}\left|D^{m} u\right|^{2} d x+C \kappa \Phi(r)+C_{\kappa} r^{n-2 m+2 \alpha}[u]_{\alpha}^{2}
\end{aligned}
$$

where we applied (7.16) with $p=2$ in the second step. For $0<j \leq k<$ $m$, the properties of $\eta$, estimates (7.2) for $g_{k}$ and Young's inequality with exponents $\frac{2 m}{2 m-k}$ and $\frac{2 m}{k}$ yield

$$
\begin{aligned}
I_{j k} \leq & C r^{j-k} \int_{B_{r}(x)} \eta^{2 m+j-k}\left|g_{k}\right|\left|D^{j} u\right| d x \\
\leq & \frac{1}{4}\left(\begin{array}{c}
m \\
2
\end{array}\right)^{-1} \int \eta^{2 m}\left|D^{m} u\right|^{2} d x+\sum_{l=1}^{m-1} \int \eta^{2 m}\left|D^{l} u\right|^{2 m / l} d x \\
& +C r^{2 m j / k-2 m} \int \eta^{2 m j / k}\left|D^{j} u\right|^{2 m / k} d x .
\end{aligned}
$$

Applying (7.15) and (7.16) with $p=\frac{2 m}{k}$ in the case $j<k$, this yields

$$
\begin{aligned}
I_{j k} \leq & \frac{1}{4}\left(\begin{array}{c}
m \\
2
\end{array}\right)^{-1} \int \eta^{2 m}\left|D^{m} u\right|^{2} d x \\
& +C\left(\kappa+\left(r^{\alpha}[u]_{\alpha}\right)^{2 / m}\right) \Phi(r)+C_{\kappa} r^{n-2 m+2 \alpha}[u]_{\alpha}^{2}
\end{aligned}
$$


Finally, we have for all $0 \leq k<m$

$$
\begin{aligned}
\mathbb{I}_{0 k} \leq & C r^{-k} \int \eta^{2 m-k}\left|g_{k}\right||u-\bar{u}| d x \\
\leq & \frac{1}{4}\left(\begin{array}{c}
m \\
2
\end{array}\right)^{-1} \int \eta^{2 m}\left|D^{m} u\right|^{2} d x \\
& +\sum_{l=1}^{m-1} \int\left(\eta^{2 m}\left|D^{l} u\right|^{2 m / l} d x+C r^{-2 m}|u-\bar{u}|^{2 m / k}\right) d x
\end{aligned}
$$

where we applied Young's inequality with exponents $\frac{2 m}{m-k}$ and $\frac{2 m}{k}$. By (7.15) and (7.14), and since $\|u-\bar{u}\|_{L^{\infty}} \leq C(N)$, this implies

$$
\mathbb{I}_{0 k} \leq \frac{1}{4}\left(\begin{array}{c}
m \\
2
\end{array}\right)^{-1} \int \eta^{2 m}\left|D^{m} u\right|^{2} d x+C\left(r^{\alpha}[u]_{\alpha}\right)^{2 / m} \Phi(r)+C r^{n-2 m+2 \alpha}[u]_{\alpha}^{2}
$$

Putting estimates (7.18) to (7.20) together, inequality (7.17) yields

$$
\begin{aligned}
\Phi\left(\frac{r}{2}\right) & \leq \int \eta^{2 m}\left|D^{m} u\right|^{2} d x \\
& \leq \frac{1}{2} \int \eta^{2 m}\left|D^{m} u\right|^{2} d x+C\left(\kappa+\left(r^{\alpha}[u]_{\alpha}\right)^{2 / m}\right) \Phi(r)+C_{\kappa} r^{n-2 m+2 \alpha}[u]_{\alpha}^{2}
\end{aligned}
$$

We absorb the first integral on the right-hand side and choose $\kappa>0$ and $0<r_{0}<1$ small enough to ensure $2 C\left(\kappa+\left(r_{0}^{\alpha}[u]_{\alpha}\right)^{2 / m}\right) \leq \varepsilon$ for some $\varepsilon<$ $2^{-(n-2 m+2 \alpha)}$. Here, $r_{0}$ may depend on $[u]_{\alpha}$. As a result, we have

$$
\Phi\left(\frac{r}{2}\right) \leq \varepsilon \Phi(r)+C r^{n-2 m+2 \alpha}[u]_{\alpha}^{2}
$$

for all $0<r<r_{0}$. By a standard iteration argument as in [8, p. 86], this implies

$$
\Phi(\rho) \leq C\left(\frac{\rho}{r_{0}}\right)^{n-2 m+2 \alpha} \Phi\left(r_{0}\right)+C \rho^{n-2 m+2 \alpha}[u]_{\alpha}^{2} \quad \text { for all } 0<\rho<r_{0} .
$$

We have thus shown that

$$
\sup _{B_{\rho}(x) \subset B_{R}} \rho^{2 m-2 \alpha-n} \int_{B_{\rho}(x)}\left|D^{m} u\right|^{2} d x<\infty \quad \text { for all } 0<R<1 .
$$


Now we will improve this Morrey space estimate with the help of Lemma 7.1. We claim that for every non-integer $\gamma=[\gamma]+\beta \in(0, m)$, we have

$$
\begin{aligned}
& u \in C^{[\gamma], \beta}(B, N) \quad \text { and } \\
& \sup _{B_{\rho}(x) \subset B_{R}} \rho^{2 m-2 \gamma-n} \int_{B_{\rho}(x)}\left|D^{m} u\right|^{2} d x<\infty \quad \text { for all } R \in(0,1) .
\end{aligned}
$$

Here, $[\gamma]$ denotes the largest integer smaller than $\gamma$. As shown above, this holds with $\gamma=\alpha \in(0,1)$. For the proof of the claim we will show that whenever the above property is satisfied for some non-integer $0<\gamma<m$, then it is also satisfied for all non-integer $\gamma_{*}<\min \left(\frac{m+1}{m} \gamma, m\right)$. We thus assume that (7.21) holds for $\gamma=[\gamma]+\beta<m$, where $0<\beta<1$. We choose some arbitrary $R \in(0,1)$ and let $S:=\frac{1}{2}(1+R)>R$. Since $u \in C^{[\gamma], \beta}(B, N)$, we know that for the Taylor polynomials $P_{x}$ of degree $[\gamma]$ at the points $x$, there holds

$$
\sum_{l=1}^{[\gamma]}\left\|D^{l} u\right\|_{L^{\infty}\left(B_{S}\right)} \leq \widetilde{C} \quad \text { and } \quad\left\|u-P_{x}\right\|_{L^{\infty}\left(B_{\rho}(x)\right)} \leq \widetilde{C} \rho^{[\gamma]+\beta}=\widetilde{C} \rho^{\gamma}
$$

for all $x \in B_{\underset{S}{C}}$ and all $0<\rho<S-|x|$. Here and in the following, constants denoted by $\widetilde{C}$ may depend on $u$ and $R$. From the Nirenberg interpolation estimates, we deduce furthermore for all $[\gamma]+1 \leq l \leq m$

$$
\begin{aligned}
& \rho^{2 m-n} \int_{B_{\rho}(x)}\left|D^{l} u\right|^{2 m / l} d x \\
& \leq C\left\|u-P_{x}\right\|_{L^{\infty}\left(B_{\rho}(x)\right)}^{2(m-l) / l} \rho^{2 m-n} \int_{B_{\rho}(x)}\left|D^{m} u\right|^{2} d x+C\left\|u-P_{x}\right\|_{L^{\infty}\left(B_{\rho}(x)\right)}^{2 m / l} \\
& \leq \widetilde{C} \rho^{2 m \gamma / l}
\end{aligned}
$$

by estimates (7.21) and (7.22), where here $x \in B_{S}$ and $\rho<S-|x|$. Using (7.22) and (7.23), we may estimate the multilinear forms $g_{k}$ from the Euler equation (7.2) as follows. For $0 \leq k \leq m-1$ and $0<\rho<S-|x|$, there holds

$$
\begin{aligned}
& \rho^{2 m-n} \int_{B_{\rho}(x)}\left|g_{k}\right|^{2 m /(2 m-k)} d x \\
& \leq C \rho^{2 m-n} \sum_{l=1}^{m} \int_{B_{\rho}(x)}\left|D^{l} u\right|^{2 m / l} d x \leq \widetilde{C} \rho^{2 m}+\widetilde{C} \sum_{l=[\gamma]+1}^{m} \rho^{2 m \gamma / l} .
\end{aligned}
$$


This means that

$$
\begin{aligned}
& \left(\rho^{2 m-n} \int_{B_{\rho}(x)}\left|g_{k}\right|^{2 m /(2 m-k)} d x\right)^{(2 m-k) / 2 m} \\
& \leq \widetilde{C} \rho^{2 m-k}+\widetilde{C} \rho^{(2 m-k) \gamma / m} \leq \widetilde{C} \rho^{m+1}+\widetilde{C} \rho^{\gamma+\gamma / m}
\end{aligned}
$$

since $k \leq m-1$. Consequently, the assumptions of Lemma 7.1 are satisfied on $B_{S}$ with the exponent $\hat{\gamma}:=\min \left(m+1, \frac{m+1}{m} \gamma\right)$. The lemma thus yields for any noninteger $\gamma_{*}<\min \left(\frac{m+1}{m} \gamma, m\right)$

$$
\sum_{\gamma_{*}<l \leq m} \rho^{2 l-n} \int_{B_{\rho}(x)}\left|D^{l} u\right|^{2} d x \leq \widetilde{C} \rho^{2 \gamma_{*}} \quad \text { for all } B_{\rho}(x) \subset B_{R} .
$$

If we write $\gamma_{*}=:\left[\gamma_{*}\right]+\beta_{*}$, this implies $u \in C^{\left[\gamma_{*}\right], \beta_{*}}\left(B_{R}, N\right)$ by the Dirichlet growth theorem. Since $0<R<1$ was arbitrary, we thus have established (7.21) for $\gamma_{*}$ instead of $\gamma$. Since it holds for $\gamma=\alpha$, we have established (7.21) for all $0<\gamma<m$.

Now let $\gamma_{*}=m+\beta$, where $0<\beta<1$ is arbitrary, and observe that for $\gamma:=\frac{m}{m+1} \gamma_{*}<m$, property (7.21) is fulfilled. As above, we deduce estimate (7.24) for this value of $\gamma$, meaning that we can apply Lemma 7.1 with $\gamma_{*}=$ $m+\beta \in(m, m+1)$. The lemma yields for any $0<\tilde{\beta}<\beta$

$$
f_{B_{\rho}(x)}\left|D^{m} u-\left(D^{m} u\right)_{x, \rho}\right|^{2} d x \leq \widetilde{C} \rho^{2 \tilde{\beta}} \quad \text { for all balls } B_{\rho}(x) \subset B_{R}
$$

where $R \in(0,1)$ is arbitrary. From Morrey's lemma we thus infer $u \in C^{m, \tilde{\beta}}$ $(B, N)$ for any $0<\tilde{\beta}<1$. The higher regularity can be deduced from classical Schauder theory [5, Theorem 2'].

\section{Intrinsically polyharmonic maps}

The intrinsic case is somewhat more technical, but it turns out that the regularity proof carries over with few modifications. The intrinsic $m$-polyenergy for mappings $u \in W^{m, 2}(\Omega, N)$ is

$$
\widetilde{E}_{m}(u)=\frac{1}{2} \int_{\Omega}\left|\nabla^{m-1} D u\right|^{2} d x
$$

where $\nabla$ denotes the covariant derivative with respect to both $M$ and $N$. 
First of all, we observe that there are $\mathbb{R}^{K}$-valued $\ell$-linear forms $A_{\mathfrak{m}}$ on $N$ for each $\mathfrak{m}=\left(m_{1}, \ldots m_{\ell}\right)$ in

$$
\mathcal{A}:=\left\{\left(m_{1}, \ldots, m_{\ell}\right): \ell \geq 2, m_{i} \geq 1 \text { for all } i, m_{1}+\cdots+m_{\ell}=m\right\}
$$

such that

$$
\nabla^{m-1} D u=D^{m} u+\sum_{\mathfrak{m} \in \mathcal{A}}\left(A_{\mathfrak{m}} \circ u\right)\left(D^{m_{1}} u, \ldots, D^{m_{\ell}} u\right)
$$

With

$$
\mathcal{B}:=\left\{\left(m_{1}, \ldots, m_{\ell}\right): \ell \geq 3,1 \leq m_{i} \leq m \text { for all } i, m_{1}+\cdots+m_{\ell}=2 m\right\}
$$

the above equation implies

$$
\left|\nabla^{m-1} D u\right|^{2}=\left|D^{m} u\right|^{2}+\sum_{\mathfrak{m} \in \mathcal{B}}\left(B_{\mathfrak{m}} \circ u\right)\left(D^{m_{1}} u, \ldots, D^{m_{\ell}} u\right)
$$

with certain $\ell$-linear forms $B_{\mathfrak{m}}$ on $N$ depending on $N$ and $\mathfrak{m}$ only. This implies that the Euler-Lagrange equation for $\widetilde{E}_{m}$ is of the form

$$
\left\langle\Delta^{m} u, \eta\right\rangle=\sum_{\mathfrak{m} \in \mathcal{B}}\left(Z_{\mathfrak{m}} \circ u\right)\left(D^{m_{1}} \eta, D^{m_{2}} u, \ldots, D^{m_{\ell}} u\right)
$$

for all vector fields $\eta \in W^{m, 2}\left(\Omega, \mathbb{R}^{K}\right)$ that are tangential along $u$, with yet another set of $\ell$-linear forms $Z_{\mathfrak{m}}$ on $N$.

Now we repeat the proof of the Euler-Lagrange equation in the form (4.4), but instead of $\left\langle\Delta^{m} u, \eta\right\rangle=0$, we use (8.1). Thus we have derived the Euler-Lagrange equation for intrinsically polyharmonic maps in the form suitable for using frames: for all tangential vector fields $\eta \in W^{m, 2}$ along $u$, we have

$$
\begin{aligned}
\Delta^{m-1} \operatorname{div}\langle D u, \eta\rangle= & \Delta^{m-1}\langle D u, D \eta\rangle+\sum_{\mathfrak{m} \in \mathcal{B}}\left(Z_{\mathfrak{m}} \circ u\right)\left(D^{m_{1}} \eta, D^{m_{2}} u, \ldots, D^{m_{\ell}} u\right) \\
& +\sum_{k=1}^{m-1} \sum_{l=0}^{k}(-1)^{k-l}\left(\begin{array}{c}
k \\
l
\end{array}\right)\left[\Delta^{m-k-1} \operatorname{div}^{l}\left\langle D^{k+1} u, D^{k-l+1} \eta\right\rangle\right. \\
& \left.+\Delta^{m-k-1} \operatorname{div}^{l+1}\left\langle D^{k} u, D^{k-l+1} \eta\right\rangle\right] .
\end{aligned}
$$

This means there are just a couple of additional terms to be handled compared to the extrinsic case. In (6.3) these are the solutions $\zeta_{\mathfrak{m}}^{i}$ of

$$
\Delta^{m} \zeta_{\mathfrak{m}}^{i}=\varphi\left(Z_{\mathfrak{m}} \circ u\right)\left(D^{m_{1}} e_{i}, D^{m_{2}} u, \ldots, D^{m_{\ell}} u\right)
$$


to be added in the sum of the left-hand side. As in (6.6) we have

$$
\left|D^{m} \zeta_{\mathfrak{m}}^{i}(x)\right| \leq C \int_{\mathbf{R}^{2 m}} \varphi(y)\left|D^{m} G(x-y)\right|\left|D^{m_{1}} e_{i}(y)\right| \prod_{i=2}^{\ell}\left|D^{m_{i}} u(y)\right| d y .
$$

Since $\ell \geq 3$, there are at least two factors involving $u$, which will be crucial in our estimates. Without loss of generality we may assume $m_{2}=$ $\max _{i=2, \ldots, \ell} m_{i}$, then $m_{i}<m$ for all $i \geq 3$. We abbreviate $\mu:=\min \{2 m-$ $\left.m_{1}-m_{2}, m-1\right\}$. Then we have

$$
\begin{aligned}
& \left|D^{m} \zeta_{\mathfrak{m}}^{i}(x)\right| \\
& \leq C \int_{\mathbf{R}^{2 m}} \varphi(y)\left|D^{m} G(x-y)\right|\left|D^{m_{1}} e_{i}(y)\right|\left|D^{m_{2}} u(y)\right| \prod_{i=3}^{\ell}\left|D^{m_{i}} u(y)\right| d y \\
& \leq C \int_{\mathbf{R}^{2 m}} \varphi(y)\left|D^{m} G(x-y)\right|\left|D^{m_{1}} e_{i}(y)\right|\left|D^{m_{2}} u(y)\right| \\
& \quad \times \sum_{k=1}^{\mu}\left|D^{k} u(y)\right|^{\left(2 m-m_{1}-m_{2}\right) / k} d y
\end{aligned}
$$

by Young's inequality. This implies

$$
\begin{aligned}
& \left\|D^{m} \zeta_{\mathfrak{m}}^{i}\right\|_{L^{2, \infty}} \\
& \quad \leq C\left\|\varphi\left|D^{m_{1}} e_{i}\right|\left|D^{m_{2}} u\right| \sum_{k=1}^{\mu}\left|D^{k} u\right|^{\left(2 m-m_{1}-m_{2}\right) / k}\right\|_{L^{1}} \\
& \quad \leq C\left\|D^{m_{1}} e_{i}\right\|_{L^{2 m / m_{1}, 2}(B)}\left\|D^{m_{2}} u\right\|_{L^{2 m / m_{2}, \infty}(B)} \sum_{k=1}^{\mu}\left\|D^{k} u\right\|_{L^{2 m / k, 2\left(2 m-m_{1}-m_{2}\right) / k}(B)}^{\left(2 m-m_{1}-m_{2}\right) / k} \\
& \quad \leq C\left\|D e_{i}\right\|_{W^{m-1,2}(B)}\left\|D^{m_{2}} u\right\|_{L^{2 m / m_{2}, \infty}(B)} \sum_{k=1}^{m-1}\left\|D^{k} u\right\|_{L^{2 m / k, 2}(B)}^{\left(2 m-m_{1}-m_{2}\right) / k} \\
& \quad \leq C \varepsilon_{0} R_{\infty} S_{2}^{\left(2 m-m_{1}-m_{2}\right) / m} \leq C \varepsilon_{0} R_{\infty}
\end{aligned}
$$

under the assumptions of Proposition 6.1, using the notation from Section 6 and the estimates $(6.14),(6.15)$. Therefore, $\left\|D^{m} \zeta_{\mathfrak{m}}^{i}\right\|_{L^{2, \infty}}$ is estimated by the same terms as $\left\|D^{m} w_{i}\right\|_{L^{2, \infty}}$ in (6.16), which means the additional terms do not spoil the proof of Proposition 6.1, and therefore of Hölder continuity, in the intrinsic case.

All that remains to be done for higher regularity is to check that also intrinsically harmonic maps satisfy an Euler equation of the form (7.2) in 
the sense of distributions. The weak form of the Euler-Lagrange equation can now be derived from

$$
\begin{aligned}
& \int\left\langle D^{m} u, D^{m}(\Pi(u) V)\right\rangle d x \\
& \quad=(-1)^{m} \sum_{\mathfrak{m} \in \mathcal{B}} \int\left(Z_{\mathfrak{m}} \circ u\right)\left(D^{m_{1}}(\Pi(u) V), D^{m_{2}} u, \ldots, D^{m_{\ell}} u\right) d x
\end{aligned}
$$

rather than (4.1). In the terms with $m_{1}=m$, there holds $m_{i}<m$ for $i=$ $2, \ldots, l$. Therefore, we may integrate by parts and may thus assume that $m_{1}<m$ in all summands. Expanding $D^{m_{1}}(\Pi(u) V)$, we find that the additional terms compared to the extrinsic case are just of the form that (7.2) allows. This completes the proof also in the intrinsic case.

\section{Acknowledgment}

We thank Melanie Rupflin for carefully reading the paper and for many comments.

\section{References}

[1] G. Angelsberg, A monotonicity formula for stationary biharmonic maps, Math. Z. 252 (2006), 287-293.

[2] G. Angelsberg and D. Pumberger, A regularity result for polyharmonic maps with higher integrability, Ann. Global Anal. Geom. 35 (2009), 63-81.

[3] J. Bramble and L. Payne, Mean value theorems for polyharmonic functions, Amer. Math. Monthly 73 (II) (1966), 124-127.

[4] S.-Y.A. Chang, L. Wang and P. Yang, A regularity theory for biharmonic maps, Comm. Pure Appl. Math. 52 (1999), 1113-1137.

[5] A. Douglis and L. Nirenberg, Interior estimates for elliptic systems of partial differential equations, Comm. Pure Appl. Math. 8 (1955), 503-538.

[6] J. Eells and L. Lemaire, Selected topics in harmonic maps, C. B. M. S. Regional Conf. Ser. Math. 50, Amer. Math. Soc., Providence, RI, 1983.

[7] A. Gastel, The extrinsic polyharmonic map heat flow in the critical dimension, Adv. Geom. 6 (2006), 501-521. 
[8] M. Giaquinta, Multiple Integrals in the Calculus of Variations and Nonlinear Elliptic Systems, Princeton University Press, Princeton, NJ, 1983.

[9] P. Goldstein, P. Strzelecki and A. Zatorska-Goldstein, On polyharmonic maps into spheres in the critical dimension, Ann. IHP, Anal. Non Linéaire, to appear.

[10] H.-C. Grunau, G. Sweers, Positivity for equations involving polyharmonic operators with Dirichlet boundary conditions, Math. Ann. 307 (1997), 589-626.

[11] T. Iwaniec and G. Martin, Quasiregular mappings in even dimensions, Acta Math. 170 (1993), 29-81.

[12] T. Lamm and T. Rivière, Conservation laws for fourth order systems in four dimensions, Comm. Partial Differential Equations 33(2) (2008), $245-262$.

[13] T. Lamm and C. Wang, Boundary regularity for polyharmonic maps in the critical dimension, Adv. Calc. Var. 2(1) (2009), 1-16.

[14] R. Moser, A variational problem pertaining to biharmonic maps, Comm. Partial Differential Equations 33(9) (2008), 1654-1689.

[15] L. Nirenberg, An extended interpolation inequality, Ann. Scuola Norm. Sup. Pisa (3) 20 (1966), 733-737.

[16] R. O'Neil, Convolution operators and $L(p, q)$ spaces, Duke Math. J. 30 (1963), 129-142.

[17] C. Scheven, Dimension reduction for the singular set of biharmonic maps, Adv. Calc. Var. 1 (2008), 53-91.

[18] J. Shatah and M. Struwe, The Cauchy problem for wave maps, Int. Math. Res. Not. 11 (2002), 555-571.

[19] R. Schoen and K. Uhlenbeck, Boundary regularity and the Dirichlet problem for harmonic maps, J. Differential Geom. 18 (1983), 253-268.

[20] E. Stein and G. Weiss, Introduction to Fourier analysis on Euclidean spaces, Princeton Math. Ser., 32, Princeton University Press, 1971.

[21] L. Tartar, Imbedding theorems of Sobolev spaces into Lorentz spaces, Boll. Unione Mat. Ital., Sez. B, Artic. Ric. Mat. 8 (1998), 479-500. 
[22] K. Uhlenbeck, Connections with $L^{p}$ bounds on curvature, Comm. Math. Phys. 83 (1982), 31-42.

[23] C. Wang, Biharmonic maps from $\mathbb{R}^{4}$ into a Riemannian manifold, Math. Z. 247 (2004), 65-87.

[24] C. Wang, Stationary biharmonic maps from $\mathbb{R}^{m}$ into a Riemannian manifold, Comm. Pure Appl. Math. 57 (2004), 419-444.

[25] W. Ziemer, Weakly differentiable functions. Sobolev spaces and functions of bounded variation, Graduate Texts in Mathematics 120, Springer, New York, 1989.

Department Mathematik Der Friedrich-Alexander-Universität

BISMARCKSTRASSE $1 \frac{1}{2}$

D-91054 ERLANGEN

GERMANY

E-mail address: gastel@mi.uni-erlangen.de; scheven@mi.uni-erlangen.de

RECEIVED July 20, 2008 